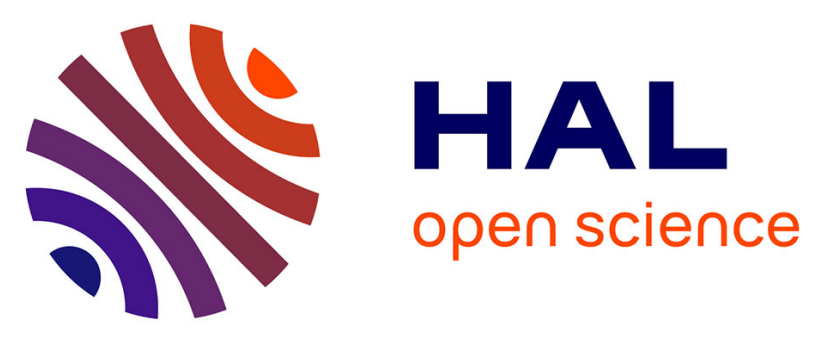

\title{
In situ observations of ions and magnetic field around Phobos: the mass spectrum analyzer (MSA) for the Martian Moons eXploration (MMX) mission
}

Shoichiro Yokota, Naoki Terada, Ayako Matsuoka, Naofumi Murata, Yoshifumi Saito, Dominique Delcourt, Yoshifumi Futaana, Kanako Seki, Micah J Schaible, Kazushi Asamura, et al.

\section{To cite this version:}

Shoichiro Yokota, Naoki Terada, Ayako Matsuoka, Naofumi Murata, Yoshifumi Saito, et al.. In situ observations of ions and magnetic field around Phobos: the mass spectrum analyzer (MSA) for the Martian Moons eXploration (MMX) mission. Earth Planets and Space, 2021, 73 (1), pp.216. 10.1186/s40623-021-01452-x . hal-03507139

\section{HAL Id: hal-03507139 \\ https://hal.sorbonne-universite.fr/hal-03507139}

Submitted on 3 Jan 2022

HAL is a multi-disciplinary open access archive for the deposit and dissemination of scientific research documents, whether they are published or not. The documents may come from teaching and research institutions in France or abroad, or from public or private research centers.
L'archive ouverte pluridisciplinaire HAL, est destinée au dépôt et à la diffusion de documents scientifiques de niveau recherche, publiés ou non, émanant des établissements d'enseignement et de recherche français ou étrangers, des laboratoires publics ou privés. 


\title{
In situ observations of ions and magnetic field around Phobos: the mass spectrum analyzer (MSA) for the Martian Moons eXploration (MMX) mission
}

\author{
Shoichiro Yokota ${ }^{*}$ (D), Naoki Terada ${ }^{2}$, Ayako Matsuoka ${ }^{3}$, Naofumi Murata ${ }^{4}$, Yoshifumi Saito ${ }^{5}$, \\ Dominique Delcourt ${ }^{6}$, Yoshifumi Futaana ${ }^{7}$, Kanako Seki $^{8}$, Micah J. Schaible ${ }^{9}, K^{\prime}$ azushi Asamura ${ }^{5}$, \\ Satoshi Kasahara ${ }^{8}$, Hiromu Nakagawa ${ }^{2}$, Masaki N. Nishino ${ }^{5}$, Reiko Nomura ${ }^{5}$, Kunihiro Keika ${ }^{8}$, Yuki Harada ${ }^{3}$ and \\ Shun Imajo ${ }^{3}$
}

\begin{abstract}
The mass spectrum analyzer (MSA) will perform in situ observations of ions and magnetic fields around Phobos as part of the Martian Moons eXploration (MMX) mission to investigate the origin of the Martian moons and physical processes in the Martian environment. MSA consists of an ion energy mass spectrometer and two magnetometers which will measure velocity distribution functions and mass/charge distributions of low-energy ions and magnetic field vectors, respectively. For the MMX scientific objectives, MSA will observe solar wind ions, those scattered at the Phobos surface, water-related ions generated in the predicted Martian gas torus, secondary ions sputtered from Phobos, and escaping ions from the Martian atmosphere, while monitoring the surrounding magnetic field. MSA will be developed from previous instruments for space plasma missions such as Kaguya, Arase, and BepiColombo/Mio to contribute to the MMX scientific objectives.
\end{abstract}

Keywords: Martian Moons eXploration (MMX), Phobos, Mars, Mass spectrum analyzer, Magnetometer

\section{Introduction}

Mars and Earth are the two terrestrial planets in the inner solar system with moons. When studying the origin and evolution of the Earth and the Moon, it is essential to treat the two bodies together as the Earth-Moon system, because lunar explorations and analyses of the returned Apollo and Luna samples and meteorites have revealed a history of Earth-Moon co-evolution (e.g., Taylor et al. 2006). The results of these studies have led to the theory resulting in a giant impact on the birth of the Moon (Canup and Asphang 2001) and the transport of

\footnotetext{
*Correspondence: yokota@ess.sci.osaka-u.ac.jp

${ }^{1}$ Osaka University, Machikaneyama-cho, Toyonaka 560-0043, Japan Full list of author information is available at the end of the article
}

the early Earth's atmosphere to the Moon (Ozima et al. 2005, 2008). Particles from Earth's atmosphere escape to space from the polar regions, guided by Earth's magnetic field (e.g., Seki et al. 2001) and some of them have been implanted inside the lunar surface materials over billions of years (Mortimer et al. 2016; Terada et al. 2017; Li et al. 2020; Wei et al. 2020; Wang et al. 2021).

The formation and evolution of Mars have also been studied from observations by both orbiters (e.g., Albee et al. 2001; Wilson et al. 2004; Jakosky et al. 2015) and landers/rovers (e.g., Grotzinger et al. 2012), and analyses of Martian meteorites (e.g., Agee et al. 2013; Borg et al. 2005). Although our understanding of Mars is improving, that of the Martian moons is greatly limited due to the lack of information obtained from spacecraft observations and analyses of meteorites 
from the moons. Similar to previous controversies about the origin of Earth's Moon, several theories have been proposed for the birth of the Martian moons that involve either the collision of large objects with Mars (e.g., Rosenblatt et al. 2016) or the capture of asteroids (e.g., Higuchi and Ida 2017). Moreover, Martian orbiter observations have suggested that the large amount of Martian atmosphere has escaped over Mars' history (e.g., Barabash et al. 2007; Jakosky et al. 2017) and can influence Phobos' surface, predominantly through oxygen ion irradiation (Nénon et al. 2019).

Because the Martian moons are still a missing piece to our understanding of the evolution of the solar system, a Martian Moons eXploration (MMX) mission is scheduled, which will conduct remote-sensing, in situ measurements and sample return similarly to Hayabusa 1 and 2 (Fujiwara et al. 2006; Watanabe et al. 2019). The science objectives are not only to reveal the origin of the Martian moons, but also to understand physical processes in the Martian environment for investigating co-evolution of the Martian-moons system (Kuramoto et al. 2021).

In the MMX mission, it is planned to perform in situ observations of ions as a key objective. Ions in the Martian environment can come from Mars' atmosphere, sputtering and stimulated desorption from the moons, photoionization of the predicted neutral torus, and the solar wind. For in situ observations of such ions, it is suitable to use ion energy mass spectrometers and magnetometers for space plasma observation missions such as Arase (Miyoshi et al. 2018) and BepiColombo/Mio (Milillo et al. 2020). Therefore, based on ion analyzer and magnetometer development experience gained from Kaguya (Yokota et al. 2005; Saito et al. 2008a, 2010; Tsunakawa et al. 2010), MMS (Pollock et al. 2016), ARASE (Yokota et al. 2017; Asamura et al. 2018; Matsuoka et al. 2018), and BepiColombo/MIO (Delcourt et al. 2009, 2016; Saito et al. 2021; Baumjohann et al. 2020), the mass spectrum analyzer (MSA) will be composed of an ion energy mass spectrometer and magnetometers for the MMX mission. The MSA instrumentation is funded by the Japan Aerospace Exploration Agency (JAXA) and is being designed and developed in a collaboration of Osaka University and Kyoto University.

In this paper, we describe the goals of the MSA science investigation as well as document the conceptual design of the MSA instrumentation. In "Scientific objectives of MSA" and "Instrumentation of MSA" sections, we describe the MSA science goals in the context of the MMX mission and the MSA instrumentation, respectively. In "Estimation of future observation" section, we present the expected observations by MSA in the Martian environment. Finally, we describe the current status of the MSA development and summarize the paper in "Summary" section.

\section{Scientific objectives of MSA}

Mars is the outermost terrestrial planet, close to the ice/ water vapor sublimation boundary, the snowline, and is in the inner and outer solar system connection region along with the asteroid main belt. It is presumed that primordial small bodies around the snowline were supplied in large quantities to the terrestrial planetary regions and played a decisive role in the establishment of the surface life environment, including the crust, oceans and atmosphere (e.g., Maruyama and Ebisuzaki 2017). Thus, the Martian moons may contain primordial materials and are key targets for investigating the material supply to the terrestrial planets (Kuramoto et al. 2021). The MMX mission is designed to accomplish two scientific major goals: (1) clarify the origins of Martian moons and constrain processes for planetary formation and material transport in the region connecting the inner and outer solar system; and (2) identify and characterize important evolutionary processes of the Mars system which consists of Mars, its moons, and the circum-Martian space (Kuramoto et al. 2021). The two goals lead to the following six medium objectives: (1.1) reveal whether Phobos originated as a captured asteroid or resulted from a giant impact; (1.2a) if Phobos is determined to be a captured asteroid, elucidate the composition and migration process of primitive materials supplied to the region of terrestrial planets and constrain the initial conditions of Martian surface evolution; (1.2b) if Phobos is determined to originate from a giant impact, elucidate giant impact and moon formation processes in the terrestrial planetary region and evaluate its influence on the early evolutionary process of Mars; (1.3) place new constraints on Deimos' origin; (2.1) obtain a basic description of the elementary processes of surface evolution for moons in the circum-Martian environment; (2.2) add new findings and constraints on the history of changes in the Martian surface; and (2.3) constrain the mechanisms of material circulation in the Martian atmosphere affecting the transitions in the Martian climate (for details, see Kuramoto et al. 2021). To accomplish the goals and objectives, the MMX mission will perform comprehensive remote-sensing and in situ observations. In addition, more than 10-g Phobos materials will be collected and delivered to Earth for detailed characterization using laboratory instrumentation (Usui et al. 2020). The MSA science investigation will address the MMX scientific goals which are related to in situ ion and magnetic field observations in the Martian environment.

The MSA observations correspond to three of the six medium objectives 1.1, 2.1 and 2.2, as summarized in 


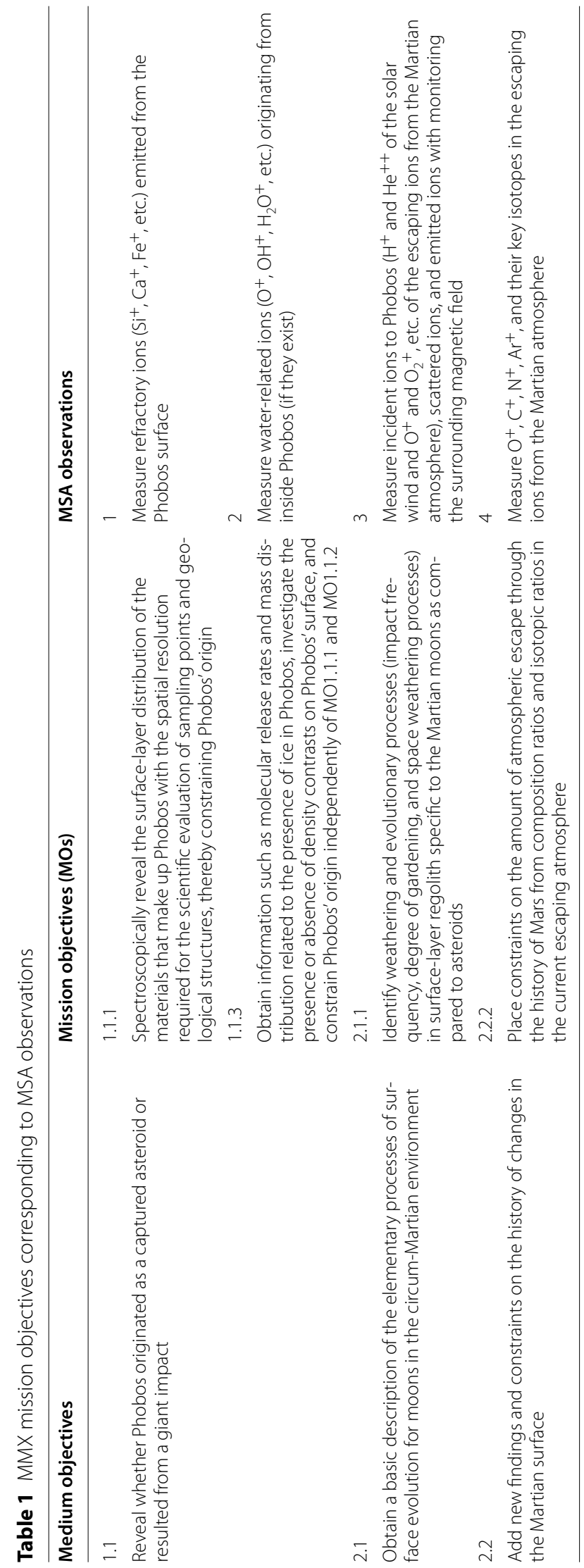


Table 1. Each medium objective is divided into different mission objectives (MOs). The MSA observations aim to accomplish the three medium objectives via four MOs, 1.1.1, 1.1.3, 2.1.1, and 2.2.2.

\section{Reveal whether Phobos originated as a captured asteroid or resulted from a giant impact (medium objective 1.1)}

The origin of the Mars moons is yet controversial, and two major theories have been proposed: primordial asteroid captures (e.g., Higuchi and Ida 2017) and in situ formation due to giant impacts (e.g., Rosenblatt et al. 2016). In the case of captured asteroid origin, the Martian moons would preserve materials which had existed in the inner and/or outer solar system during the early solar system epoch (> several $10^{7}$ years). In the other case, the Martian moons would provide the second example of the giant impact following the Earth-Moon system and would substantially contain materials of the Martian origin. Therefore, either origin of the Martian moons revealed by the MMX mission will place constraints on not only the initial condition of the Mars-moons system, but also the planet-forming processes and/or material transport in the connection region between the inner and outer solar system (Kuramoto et al. 2021).

Among the medium objective 1.1, MSA provides two observations which correspond to MO1.1.1: spectroscopically reveal the surface-layer distribution of the materials that make up Phobos with the spatial resolution required for the scientific evaluation of sampling points and geological structures, thereby constraining Phobos' origin, and MO1.1.3: obtain information such as molecular release rates and mass distribution related to the presence of ice in Phobos, investigate the presence or absence of density contrasts on Phobos' surface, and constrain Phobos' origin independently of MO1.1.1 and MO1.1.2. MSA will measure refractory ions emitted from the Phobos surface (MSA observation 1), and water-related ions originating from the inside Phobos (MSA observation 2), for MO1.1.1 and MO1.1.3, respectively (see Table 1). It should be noted that, as will be explained in detail in "Estimation of future observation" section, the measurement of the magnetic field places constraints on the observed ions being of lunar origin (Yokota et al. 2009).

\section{MSA observation 1: refractory ions from the Phobos surface}

The chemical composition of meteorites and returned samples provides various insights into the origin and evolution of their parent bodies (e.g., Saal et al. 2008; Terada et al. 2018). According to the database on the elemental abundance of meteorites, the composition ratios of several elements can be used to discriminate between different meteorite groups (Nittler et al. 2004). Thus, remote-sensing measurements of the chemical composition were made by orbiters equipped with X-ray and gamma-ray spectrometers in several solar system explorations (e.g., Feldman et al. 1998; Nittler et al. 2011; Lawrence et al. 2013), and are planned in the MMX mission using gamma-ray and neutron instruments (Lawrence et al. 2019).

When analyzing samples in the laboratory for the chemical composition, secondary ion mass spectrometry (SIMS) has been frequently used. In SIMS analysis, secondary ions are ejected from targeted samples by a primary ion beam and subsequently collected and analyzed by a mass spectrometer. In the case of small bodies which have no thick atmosphere, the solar wind directly impacts their surface and sputters secondary ions, similarly to SIMS analysis. Fluxes of the secondary ions produced by solar wind sputtering are sufficiently large $\left(\geq \sim 10^{4}\right.$ ions $\mathrm{cm}^{-2} \mathrm{~s}^{-1}$ ) to be measured by standard spaceborne ion mass spectrometers (Elphic et al. 1991; Schaible 2014; Dukes and Baragiola 2015). This means that ion observations by orbiters can be utilized for the SIMS analysis of small bodies (Johnson and Baragiola 1991; Yokota and Saito 2005). Lunar orbiters actually discovered such ions around the Moon (e.g., Yokota et al. 2009; Halekas et al. 2013). Because background sources of refractory ions are negligible, the measurement of such ions can be a robust method.

Since the density of the solar wind near Mars at $\sim 1.5$ Astronomical Unit (AU) is around half of that near the Earth and Moon, the total secondary ion flux from Phobos is expected to be $\sim 10^{4}$ ions $\mathrm{cm}^{-2} \mathrm{~s}^{-1}$ assuming the ion emission is proportional to the solar wind flux. Estimates of the sputtered secondary ion fluxes due to the solar wind and Martian magnetosphere ion sputtering are shown for a suite of Martian meteorite and carbonaceous chondrite compositions in Fig. 1. The total sputtering yields were estimated using SDTrimSP (Static-Dynamic Transportation of ion in material for Sequential and Parallel computer) (Mutzke et al. 2011), a binary collision Monte Carlo simulation program, where the incident ion energies were sampled from $\mathrm{H}^{+}, \mathrm{O}^{+}$, and $\mathrm{O}_{2}{ }^{+}$ion energy distributions derived from STATIC observations of magnetospheric ion fluxes during a single MAVEN pass through the magnetotail at Phobos orbital distances (J. Halekas, personal communication). Secondary ion fluxes were determined using an experimentally calibrated correction factor for the relative secondary ion yields (Schaible et al. 2017).

Because the ion fluxes in the magnetotail can be highly variable (Brain et al. 2015; Halekas et al. 2017), the sputtered secondary ion fluxes may vary significantly depending on the instantaneous plasma conditions. Based on ion fluxes determined from long-term MAVEN observations in the Martian system, it is expected that, on 

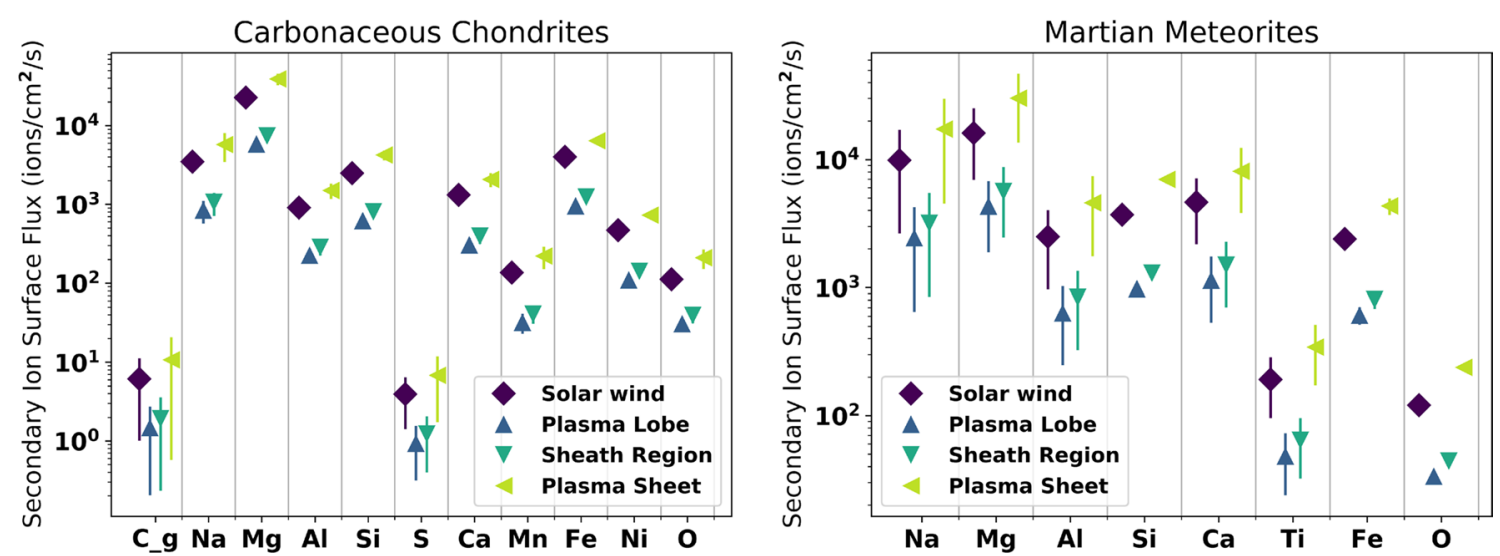

Fig. 1 Secondary ion ratios for several types of meteorites considering the solar wind and Martian magnetospheric ion sputtering. Solar wind fluxes were calculated assuming $95 \% \mathrm{H}^{+}$and $5 \% \mathrm{He}^{++}$ions, and incident ion fluxes for the various magnetosphere regions were determined from MAVEN STATIC observations combined with SDTrimSP sputtering simulations and experimental calibration of relative secondary ion yields (Schaible et al. 2017)

average, surface sputtering is slightly enhanced in the sheath region and lower in the plasmasheet (Nénon et al. 2019). However, it is clear that significant sputtered ion fluxes are expected at nearly all points in the Phobos and Deimos orbits. This is especially important due to the fact that Phobos is tidally locked to Mars so that the two hemispheres see distinctly different ion irradiation environments. The Mars-facing hemisphere is exposed to predominantly oxygen ions while the anti-Mars hemisphere is exposed to solar wind (Nénon et al. 2019), and thus distinguishing between magnetospheric oxygen and oxygen from water ice is critical. Although, due to its high electronegativity, oxygen is rarely sputtered as a positive ion, it can be easily measured in negative ion channels. Classification of targeted small bodies can be carried out by measuring the flux ratios of refractory ions such as $\mathrm{Mg}^{+}, \mathrm{Ca}^{+}$, and $\mathrm{Fe}^{+}$to $\mathrm{Si}^{+}$even with an accuracy of $\sim 50 \%$ (Schaible et al. 2017). Therefore, MSA measurements of secondary ions sputtered from Phobos by the solar wind (especially refractory ions) will allow us to discriminate between the formation models.

\section{MSA observation 2: water-related ions from the Phobos interior}

To further constrain the origin of the Martian moons independently of observations of surface materials and analyses of returned samples, the MMX missions will examine their internal structures, especially the existence of ice. Captured primordial asteroids would preserve a large amount of ice inside them, while giant impacts cause depletion of volatile elements including water similarly to the Moon (e.g., Canup and Asphang 2001). In the case of the captured asteroid origin, the current $\mathrm{H}_{2} \mathrm{O}$ release rate of $\sim 0.3$ to $\sim 3 \mathrm{~g} / \mathrm{s}\left(\sim 10^{22}\right.$ to $\sim 10^{23}$ molecules $\left./ \mathrm{s}\right)$ was calculated depending on the pore size and porosity by a model of the evolution of the water regime for Phobos (Fanale and Salvail 1990). Re-accreted material would be volatile-depleted, but Phobos could still emit $\mathrm{H}_{2} \mathrm{O}$ at a rate less than $\sim 10^{20}$ molecules/s, assuming that all oxygen is released as $\mathrm{H}_{2} \mathrm{O}$. This water could be caused/supplied by reactions of solar wind hydrogen with refractory oxygen in the surface rocks, escaping ions from the Martian atmosphere (Benna et al. 2015), and micrometeoroid impact (Cipriani et al. 2011; Poppe and Curry 2014), Therefore, an $\mathrm{H}_{2} \mathrm{O}$ flux of $\sim 10^{22}$ molecules/s is an indicator of the presence or absence of ice in Phobos.

Released $\mathrm{H}_{2} \mathrm{O}$ molecules can easily escape the orbit of Phobos, and become trapped in a keplerian orbit around Mars (Ip and Banaszkiewicz 1990), forming an envelope of all the permitted trajectories, or 'torus' (Krymskii et al. 1992; Poppe et al. 2016). Peak densities of $10^{4}-10^{5}$ molecules $/ \mathrm{cm}^{3}$ were derived from a model of the $\mathrm{H}_{2} \mathrm{O}$-related molecular gas torus (Mura et al. 2002) using the release rate of $10^{23}$ molecules/s. In the Phobos- 2 observation, electromagnetic ion beam waves were observed, suggesting the existence of a Phobos neutral gas torus (Baumgärtel et al. 1998).

The existence of ice in Phobos is expected to result in the $\mathrm{H}_{2} \mathrm{O}$ emission of $\sim 10^{22}$ to $\sim 10^{23}$ molecules/s and formation of the $\mathrm{H}_{2} \mathrm{O}$ gas torus of $10^{4}-10^{5}$ molecules $/ \mathrm{cm}^{3}$ (Mura et al. 2002). In the case of the $\mathrm{H}_{2} \mathrm{O}$ emission rate of $10^{22}$ molecules /s, the peak flux of $\mathrm{H}_{2} \mathrm{O}$-related ions $\left(\mathrm{O}^{+}\right.$, $\mathrm{OH}^{+}, \mathrm{H}_{2} \mathrm{O}^{+}$, etc.) of $\sim 10^{5}$ ions $\mathrm{cm}^{-2} \mathrm{~s}^{-1}$ can be estimated (Poppe et al. 2016). Such ion fluxes can be observed by standard spaceborne ion energy mass analyzers designed for measuring ions of $10^{4}-10^{8}$ ions $\mathrm{cm}^{-2} \mathrm{~s}^{-1}$ in the solar 
wind and planetary magnetosphere (e.g., Yokota et al. 2005, 2017). It should be noted that, during the solar minimum, no signatures related with such a Phobos torus were observed by Mars Express (Futaana et al. 2010a) and Mars Global Surveyor (Øieroset et al. 2010). If Phobos contains a large amount of ice, the observation of waterrelated ions by MSA can determine that its origin is a captured primordial asteroid.

\section{Obtain a basic description of the elementary processes of surface evolution for moons in the circum-Martian environment (medium objective 2.1)}

The surfaces of small bodies experience changes in their chemical composition and reflection spectrum, called 'space weathering' (Chapman 2004), caused by the impact of micrometeoroids (Sasaki et al. 2001) and solar wind ions (Vernazza et al. 2009; Matsumoto et al. 2020). The presence of planet Mars affects the frequency and velocity of meteorite and micrometeoroid impacts onto the Martian moons (Borin et al. 2017), and escaping ions from the Martian atmosphere as well as solar wind ions collide with their surfaces (Nénon et al. 2019). The nearside of Phobos, the rotation of which is currently synchronized with its revolution, might contain much more particles from the Martian atmosphere (Nénon et al. 2021). Therefore, the space weathering of the Martian moons is probably different from that of other moons and asteroids in the main belt.

\section{MSA observation 3: incident ions to Phobos, scattered ions, emitted ions, and the magnetic field}

To achieve medium objective 2.1, the MMX mission has the following task, MO2.1.1: identify weathering and evolutionary processes (impact frequency, degree of gardening, and space weathering processes) in surface-layer regolith specific to the Martian moons as compared to asteroids. To address MO2.1.1, MSA has observation 3: measure incident ions to Phobos, scattered ions, and emitted ions (see Table 1).

The solar wind typically has a flux of $\sim 10^{8}$ particles $\mathrm{cm}^{-2} \mathrm{~s}^{-1}$ composed of ions (mostly $\mathrm{H}^{+}$and $\mathrm{He}^{++}$) and electrons, sometimes varying from $10^{-1}$ to 10 times, with a magnetic field of $\sim 1$ to $\sim 10 \mathrm{~s} \mathrm{nT}$ around Mars (Trotignon et al. 1996). When the Martian moons are downstream of the solar wind relative to Mars, they are substantially shielded from the solar wind ions, but are exposed to escaping ions from the Martian atmosphere $\left(\mathrm{O}^{+}, \mathrm{O}_{2}^{+}\right.$, etc.) of $\sim 10^{4}$ to $\sim 10^{7}$ ions $\mathrm{cm}^{-2} \mathrm{~s}^{-1}$ (e.g., Lundin et al. 2004; Dong et al. 2015; Ramstad et al. 2017). In the case of the Earth's Moon, $\sim 0.1$ to $\sim 1 \%$ of incident solar wind protons are back-scattered as still protons (Saito et al. 2008b), 20\% as neutral hydrogen atoms
(McComas et al. 2009; Wieser et al. 2009), while the rest are probably injected into the lunar surface. The backscattering of the solar wind was also observed around Phobos (Futaana et al. 2010a). The solar wind not only bombards the dayside surface of the Moon, but also intrudes the wake region because of electromagnetic effects (Futaana et al. 2010b; Nishino et al. 2009, 2010, 2013; Dhanya et al. 2017). In addition, some of the solar wind is reflected in the Earth's bow shock and irradiates the nightside of the Moon (Nishino et al. 2017).

Since the Moon has large and wide magnetic anomalies (e.g., Richmond and Hood 2008; Tsunakawa et al. 2015), which deflect and/or reflect the solar wind (Saito et al. 2012), some areas are substantially shielded from the solar wind by an induced electric field (Futaana et al. 2013). Such a shielding from the solar wind can reduce the progression of the space weathering (Kramer et al. 2011; Bamford et al. 2016). Although there is no unambiguous evidence for the existence of magnetic anomalies on Phobos (Mordovskaya et al. 2001; Veselovsky 2004), their electromagnetic effects would have a significant influence on the space weathering if they exist.

As described in "MSA observation 1: refractory ions from the Phobos surface" section, secondary ions are emitted by the solar wind sputtering that affects evolutionary processes of the surface regolith layer. The ion emissions for various species were estimated at $\sim 10^{4}$ ions $\mathrm{cm}^{-2} \mathrm{~s}^{-1}$ around Phobos (Poppe and Curry 2014). In the case of the Earth's Moon, secondary ion emissions of $\sim 10^{4}$ ions $\mathrm{cm}^{-2} \mathrm{~s}^{-1}$ were estimated (Yokota and Saito 2005) and were actually observed (e.g., Yokota et al. 2009; Tanaka et al. 2009) for a variety of ions, preferably volatile species (Yokota et al. 2014, 2020). To reveal how much ions go toward and come from the Martian moons for each ion species, MSA will measure solar wind ions (mostly $\mathrm{H}^{+}$and $\mathrm{He}^{++}$), escaping ions from the Martian atmosphere $\left(\mathrm{O}^{+}, \mathrm{O}_{2}^{+}\right.$, etc.), back-scattered solar wind ions, and secondary ions emitted from the Martian moons. In addition, MSA will also measure a magnetic field to investigate the surrounding electromagnetic effects and to search for magnetic anomalies on the Martian moons.

\section{Add new findings and constraints on the history of changes in the Martian surface (medium objective 2.2)} The Martian moons could contain substantial materials of Martian origin which they have carried since their birth via ejecta (Hyodo et al. 2019) and escaping ions (e.g., Inui et al. 2018; Nénon et al. 2019). The heavy isotope enrichment in major elements $(\mathrm{C}$, $\mathrm{O}$, etc.) in the lower Martian atmosphere measured by the Curiosity rover supported the hypothesis of substantial atmospheric 
escape (Mahaffy et al. 2013). Thus, in the MMX mission, the information on the history of the Mars surface environment evolution can be provided by the analysis of returned samples (Usui et al. 2020). In addition, the MSA ion observations will evaluate not only the current status of the Martian atmospheric escape, but also will constrain its history using the isotope ratios (e.g., Jakosky et al. 2017).

\section{MSA observation 4: escaping ions from the Martian atmosphere and their key isotopes}

To contribute to the accomplishment of medium objective 2.2, MSA has observation 4: measure $\mathrm{O}^{+}, \mathrm{C}^{+}, \mathrm{N}^{+}$, $\mathrm{Ar}^{+}$, and their key isotopes in the escaping ions from the Martian atmosphere, which corresponds to one of the mission objectives, MO2.2.2: place constraints on the amount of atmospheric escape through the history of Mars from composition ratios and isotopic ratios in the current escaping atmosphere (see Table 1).

In the escape processes of the Martian atmosphere, the lighter species are preferentially removed, resulting in a remaining atmosphere enriched in the heavier isotopes (e.g., McElroy and Yung 1976; Chassefiere and Leblanc 2004). Ignoring the supply and loss of materials other than atmospheric escape, such as exchange with the surface and crust, the amount of the Martian atmosphere $N(t)$ for each species at time $t$ is expressed by a simplified equation (Chassefiere and Leblanc 2004):

$$
N(t)=N_{0} r(t)^{1 /(f-1)},
$$

where $N_{0}, r(t)$, and $f$ indicate the initial amount, isotope abundance ratio at normalized by the initial one, and isotope fractionation factor, respectively. To estimate $r(t)$, the current and initial isotope abundance ratios in the Martian atmosphere are derived from the observation data by the Curiosity rover and the analysis of primordial chondrites, respectively. Because $f$ is determined by the isotope abundance ratio at the exobase, the MAVEN observation estimated $f$ for ${ }^{38} \mathrm{Ar} /{ }^{36} \mathrm{Ar}$ and then calculated $N_{0}$, suggesting that $66 \%$ of the atmospheric argon has been lost to space (Jakosky et al. 2017). In the MMX mission, $f$ for ${ }^{18} \mathrm{O} /{ }^{16} \mathrm{O},{ }^{13} \mathrm{C} /{ }^{12} \mathrm{C}$, etc., will be derived from the MSA observation of escaping atmospheric ions.

Previous observations and computations using a model of the upper atmosphere estimated $f$, while the estimate had a large $(\sim 400 \%)$ variation for ${ }^{18} \mathrm{O} /{ }^{16} \mathrm{O}$ (e.g., McElroy and Yung 1976; Fox and Hac 2010). Thus, the estimate of $f$ within a $50 \%$ accuracy is needed in the MMX mission for further constraints on the total loss amounts of the Martian atmosphere.

\section{Instrumentation of MSA}

The MSA instrument is composed of an ion energy mass spectrometer, two magnetometers, and electronics (see Fig. 2). The ion analyzer measures distribution functions and mass distributions of low-energy $(<\sim 10 \mathrm{~s} \mathrm{keV})$ ions. The magnetometers measure the magnetic field of the solar wind which is sometimes perturbed by Mars and possibly by Phobos. The combination of ion and magnetic field sensors will allow us to measure ions emitted from Phobos and its torus as well as escaping ions from the Martian atmosphere with monitoring the solar wind to address the MMX science goals.

\section{MSA ion energy mass spectrometer}

The MSA ion energy mass spectrometer employs nearly the same measurement techniques as that of Ion energy Mass Analyzer (IMA) for the Kaguya mission (Yokota et al. 2005; Saito et al. 2008a, 2010) and mass spectrum analyzer (MSA) for BepiColombo/MIO (Delcourt et al. 2009, 2016; Saito et al. 2021). Figure 3 shows a cross section of an engineering model of the MSA ion energy mass analyzer. The ion analyzer is cylindrically symmetric in shape and consists of an energy analyzer and a mass analyzer. The aperture of $360^{\circ}$ near the sensor top and neighboring angular scanning deflectors provide a $\sim 2 \pi$ steradian field-of-view (FOV) (Yokota et al. 2005). The two angular scanning deflectors are alternately applied with a sweeping high voltage up to $+5 \mathrm{kV}$ for such a wide FOV. The energy analyzer measures energy/charge $\frac{E}{q}$ using a top-hat electrostatic method (e.g., Carlson et al. 1982) in which the inner spherical electrode is applied with a sweeping negative high voltage. In the mass analyzer, mass/charge $\frac{m}{q}$ is measured by a time-of-flight (TOF) method that uses a linear-electric field (LEF) for the higher mass resolution (e.g., McComas and Nordholt 1990). At the entrance of the mass analyzer, ultra-thin carbon foil is mounted on a metal grid to emit secondary electrons for start signals. The TOF chamber is longer than that of the previous analyzers and is optimized to achieve a high mass resolution $\left(\frac{m}{\Delta m}>100\right)$ (Gilbert et al. 2010). Both ends of the TOF chamber are supplied with static high voltage up to $\pm \sim 12 \mathrm{kV}$ for a post-acceleration and reflection of incident ions, respectively. The incident ions are detected by a micro-channel plate (MCP) assembly at the bottom as stop signals if they are neutralized by the carbon foil. In the other case, the incident ions pass through the carbon foil as ions and then are reflected by the LEF, resulting in ejection of secondary electrons at the ceiling of the TOF chamber. The secondary electrons are attracted by the LEF and are also detected by the MCP assembly as stop signals. The MCP assembly has a circular delay line anode to obtain the start signal and $360^{\circ}$-position information from the 


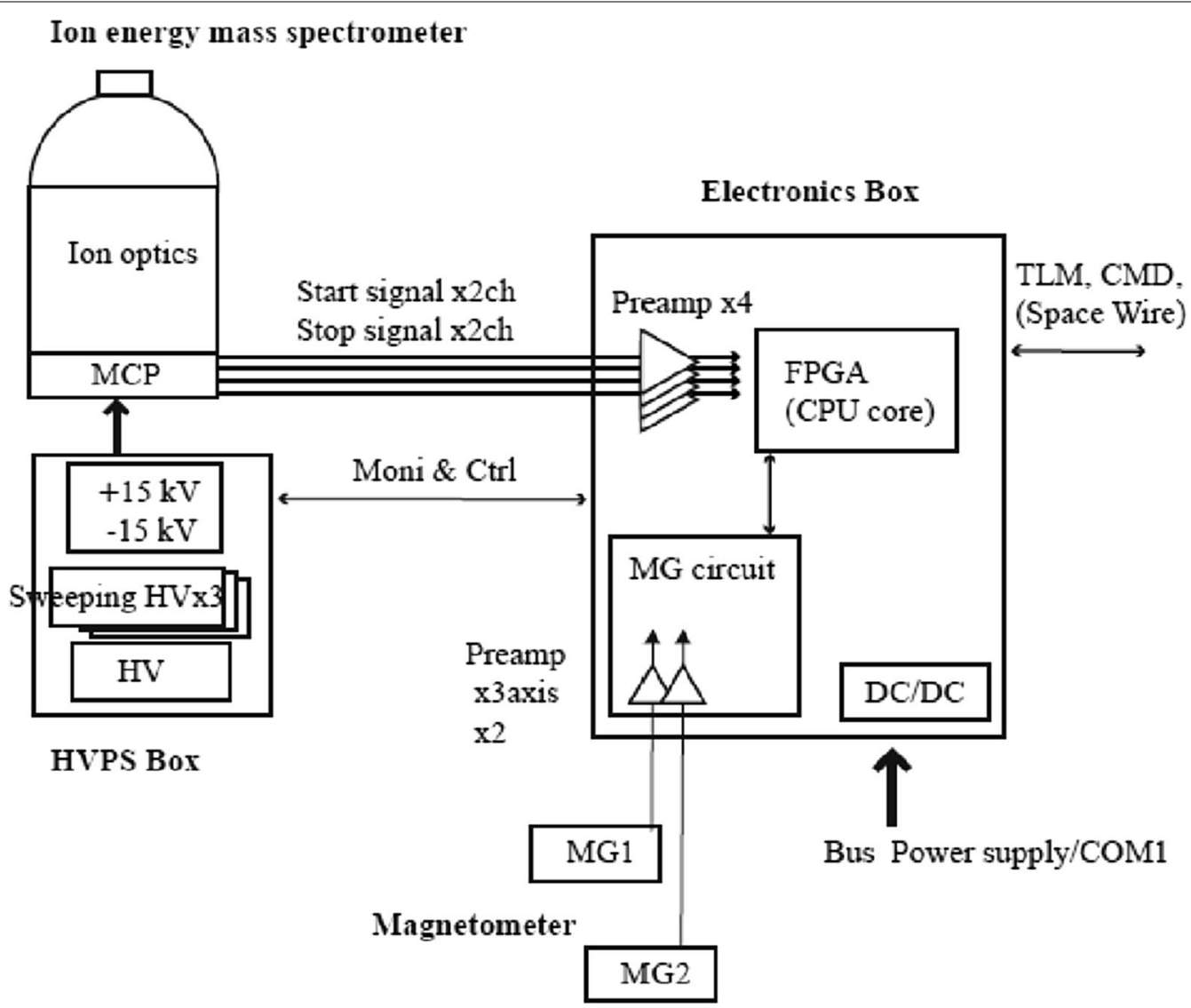

Fig. 2 Block diagram of MSA

detection of the secondary electrons emitted from the carbon foil (Saito et al. 2017). The specifications of the MSA ion analyzer are listed in Table 2.

\section{MSA magnetometer}

A fundamental mode orthogonal fluxgate (FM-OFG) technique is used for the two magnetometers (MG1 and 2) which are the components of MSA. FM-OFG was firstly proposed by Sasada (2002) and successively 'bias switching' method of the sensor excitation was designated to drastically reduce the offset in the output signal (Sasada and Usui 2003; Koga and Sasada 2003). Recently the sensor and circuit design to apply FM-OFG to space missions has been developed by improving the offset stability and noise characteristics (Murata et al. 2018, 2019). FM-OFG technique achieves significantly downsized and lightened sensor in comparison with the conventional parallel fluxgate magnetometers which have been generally used for space missions. FM-OFG is much effective to reduce the power to excite the sensor as well.

Each of two MSA magnetometers measures three orthogonal components of the magnetic field. Figure 4 shows the schematic configuration of the magnetometer with a single sensor head, while the actual sensor unit has three heads whose axes are arranged orthogonally. In the sensor head part, a couple of amorphous wire cores are strained in parallel along a resin bobbin of rod shape. The tips of the cores are connected through the copper bonding, and the roots are connected to the excitation circuit in the electronics part implemented in the MSA electronics box. The excitation circuit directly applies the Direct Current (DC)-biased alternating current (AC) excitation current, which is sinusoidal $\mathrm{AC}(f=74 \mathrm{kHz})$ current superposed on DC (bias) current, to the core. The polarity of the excitation current is periodically flipped (bias switching). The signal of the same $f \mathrm{~Hz}$ induced in the pickup coil wound around the bobbin is detected by the electronics part. More details about the FM-OFG technique using bias switching are described in Murata et al. (2018) and Murata et al. (2019). The specifications of the MSA magnetometer are listed in Table 3.

\section{MSA electronics box}

The MSA instrument includes an electronics box which is installed inside the spacecraft structure. Each analog signal from the MSA ion analyzer and magnetometers 


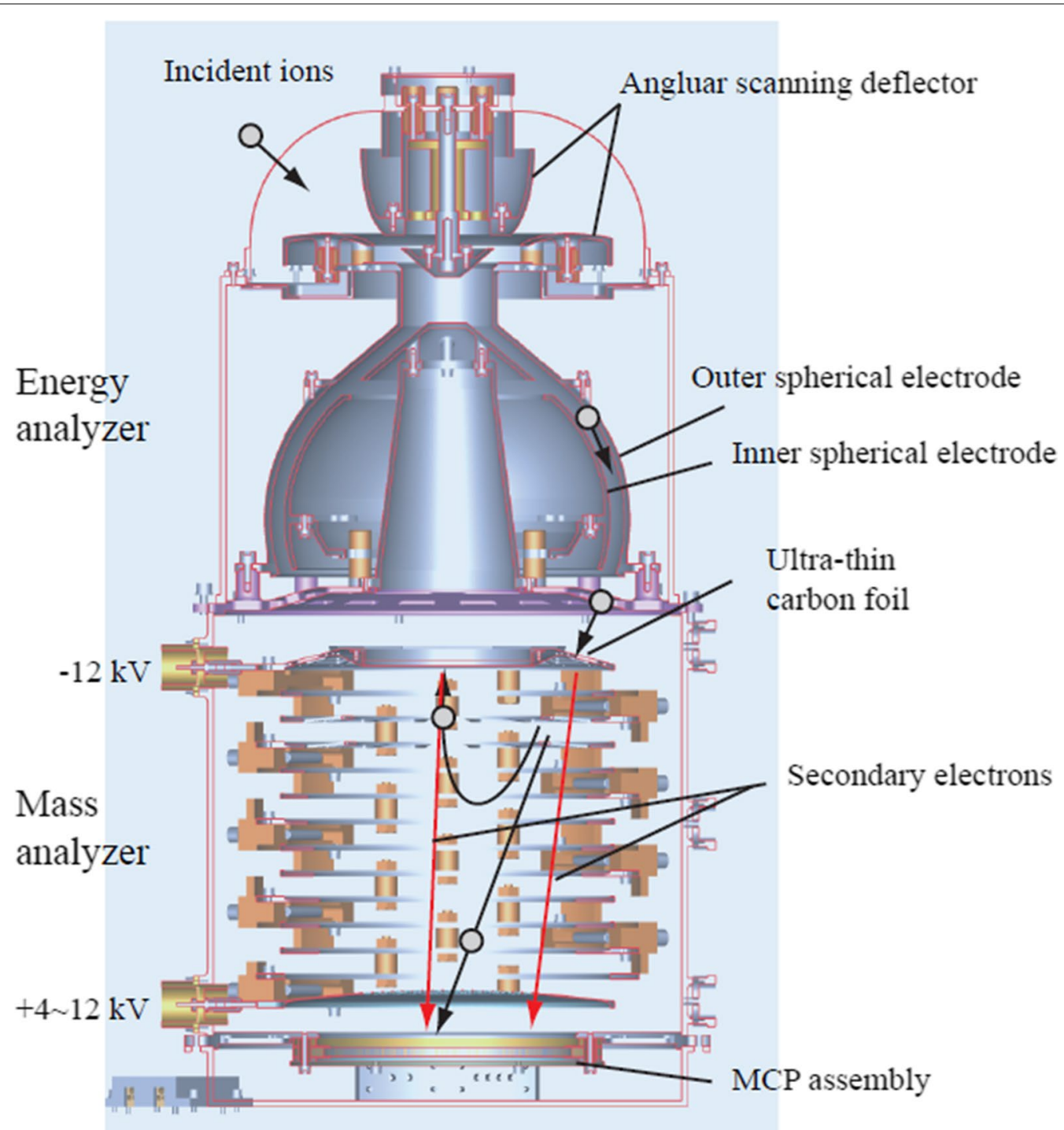

Fig. 3 Cross-sectional view of the ion optics and MCP assembly in the MSA ion energy mass spectrometer engineering model. Trajectories of incident ions (black) and secondary electrons (red) are shown

Table 2 Specifications of the MSA ion energy mass spectrometer

\begin{tabular}{|c|c|c|}
\hline Parameters & Value & Notes \\
\hline Energy range & $\sim 5$ to $\sim 30 \mathrm{k} \mathrm{eV} / \mathrm{q}$ & \\
\hline $\begin{array}{l}\text { Energy resolu- } \\
\text { tion }\end{array}$ & $\sim 10 \%$ & $\Delta E / E(F W H M)$ \\
\hline FOV range & $\geq 2 \pi s r$ & \\
\hline FOV resolution & $22.5^{\circ} \times 12.25^{\circ}$ per channel & FWHM \\
\hline Mass range & $1-100 \mathrm{amu}$ & \\
\hline Mass resolution & $>100$ & $m / \Delta m(\mathrm{FWHM})$ \\
\hline Geometric factor & $\geq 10^{-4} \mathrm{~cm}^{2} \mathrm{sr} \mathrm{eV} / \mathrm{eV}$ per channel & $\begin{array}{l}\text { Calculated in the } \\
\text { numerical model }\end{array}$ \\
\hline
\end{tabular}

is collected by the electronics box via each pre-amplifier (see Fig. 2). The signal processing and data allocation for the ion analyzer and magnetometers are made by a FieldProgrammable Gate Array (FPGA) with Central Processing Unit (CPU) cores. The FPGA also monitors and controls High Voltage Power Supplies (HVPSs) that are mounted near the ion analyzer. Telemetry data (TLM) and commands (CMDs) are transmitted and are received via the space wire interface to the spacecraft system. The electronics box has a DC-DC converter (DC/DC) to provide $\pm 12 \mathrm{~V},+5 \mathrm{~V}$, and $+3.3 \mathrm{~V}$ to each electronic board. 


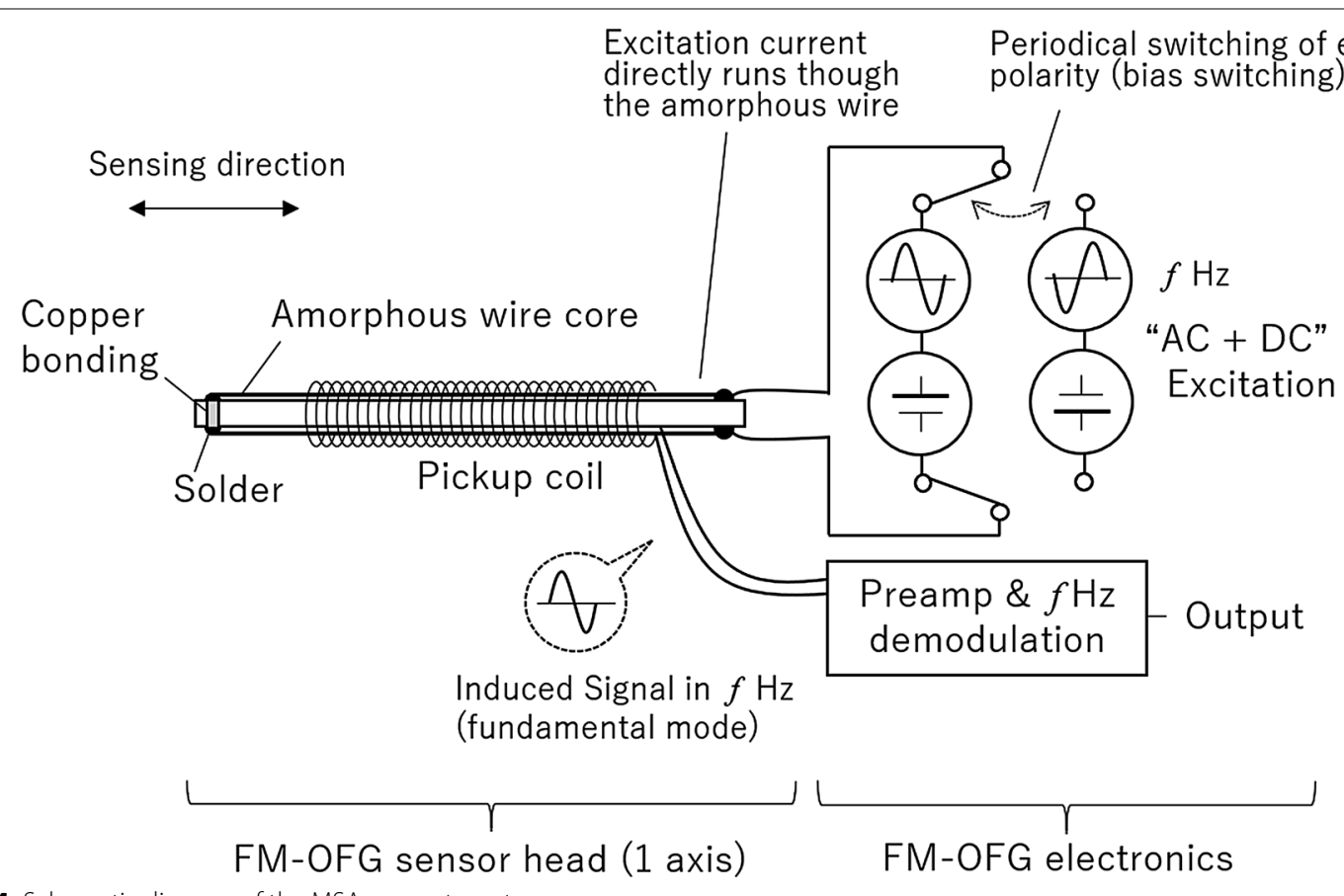

Fig. 4 Schematic diagram of the MSA magnetometer

\section{Estimation of future observation}

The MSA ion spectrometer employs the techniques used for previous ion analyzers for space plasma observations, with measured fluxes ranging from $\sim 10^{8}$ ions $\mathrm{cm}^{-2} \mathrm{~s}^{-1}$ (for solar wind ions) to $\geq \sim 10^{4}$ ions $\mathrm{cm}^{-2} \mathrm{~s}^{-1}$ (for planetary magnetospheric ions). Thus, MSA has sufficient performance to measure secondary ions of $\sim 10^{4}$ ions $\mathrm{cm}^{-2} \mathrm{~s}^{-1}$ from Phobos (MSA observation 1), water-related ions of $\sim 10^{7}$ ions $\mathrm{cm}^{-2} \mathrm{~s}^{-1}$ originating from the Phobos torus if exists (MSA observation 2), solar wind ions of $\sim 10^{8}$ ions $\mathrm{cm}^{-2} \mathrm{~s}^{-1}$ and those scattered at the Phobos surface of $10^{5}-10^{6}$ ions $\mathrm{cm}^{-2} \mathrm{~s}^{-1}$ (MSA observation 3), and escaping ions from the Martian atmosphere of $\sim 10^{4}$ to $\sim 10^{7}$ ions $\mathrm{cm}^{-2} \mathrm{~s}^{-1}$ (MSA observation 4). Compared to previous ion analyzers for Mars missions (e.g., Barabash et al. 2004; Wilson 2004;

Table 3 Specifications of the MSA magnetometers

\begin{tabular}{lll}
\hline Parameters & Value & Notes \\
\hline Dynamic range & $\pm 8000 \mathrm{nT} / \pm 60000 \mathrm{nT}$ & Switched by command \\
Resolution & $0.1 \mathrm{nT} / 0.8 \mathrm{nT}$ & \\
Sampling rate & $1 \mathrm{~Hz}$ & Nominal observation \\
& $128 \mathrm{~Hz}$ & Checkout mode \\
Noise level & $<15 \mathrm{pT} / \sqrt{\mathrm{Hz}}$ & At $1 \mathrm{~Hz}$ \\
Offset stability & $<0.027 \mathrm{nT} /{ }^{\circ} \mathrm{C}$ & \\
\hline
\end{tabular}

McFadden et al. 2015), only the mass resolution will be improved to $\mathrm{M} / \Delta \mathrm{M} \geq \sim 100$ to clearly discriminate heavy ions and their isotope from each other. Therefore, successful investigations by MSA mainly depend on the configuration and period of the observation.

\section{MSA ion observation}

During the nominal scientific observation period, the MMX spacecraft will be in a quasi-satellite orbit, orbiting Mars together with Phobos at a distance of $\sim 20$ to $\sim 200 \mathrm{~km}$ from each other (Nakamura et al. 2021). Figure $5 \mathrm{a}, \mathrm{b}$ shows the MSA observation configurations, focusing on the relations with Phobos/Mars in the Phobos/Mars-centric Solar Ecliptic coordinates, respectively. The Phobos/Mars-centric Solar Ecliptic coordinate system has the $X$-axis pointing from Phobos/Mars towards the Sun, the $Z$-axis parallel to the ecliptic northern pole, and the $Y$-axis determined in the right-handed system. When the spacecraft is in the neighborhood of Phobos, the hemispherical FOV of MSA can capture both solar wind ions and those scattered at the Phobos surface in most situations. Because the spacecraft orbits along to the Martian torus, water-related ions generated from the torus are easily observed if such a gas torus exists. The observations of water-related ions are suitable in the dayside of Mars because it is straightforward to distinguish between ions from the torus and those from the Martian atmosphere. When the spacecraft and Phobos are behind 


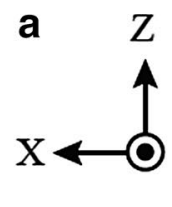

$\mathrm{Y}$

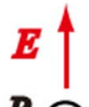

$B$ ○

$\mathrm{S} / \mathrm{C}$

SW

SW

SW

SW
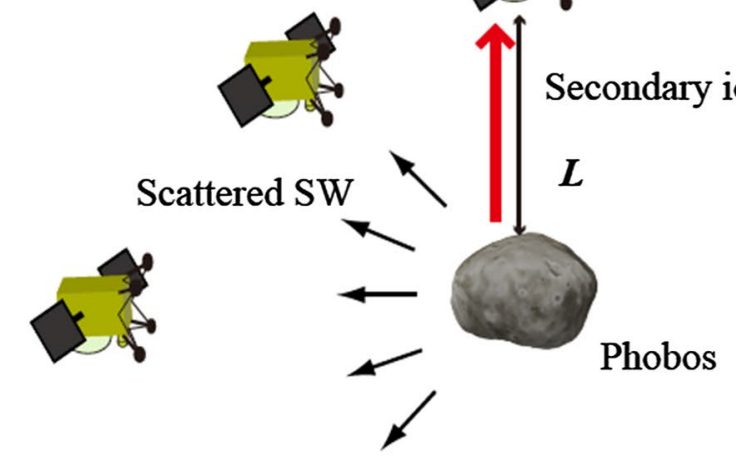

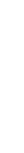

Scattered SW

SW

b

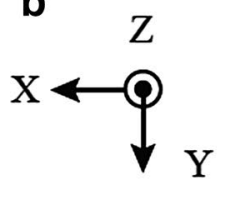

SW

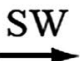

SW
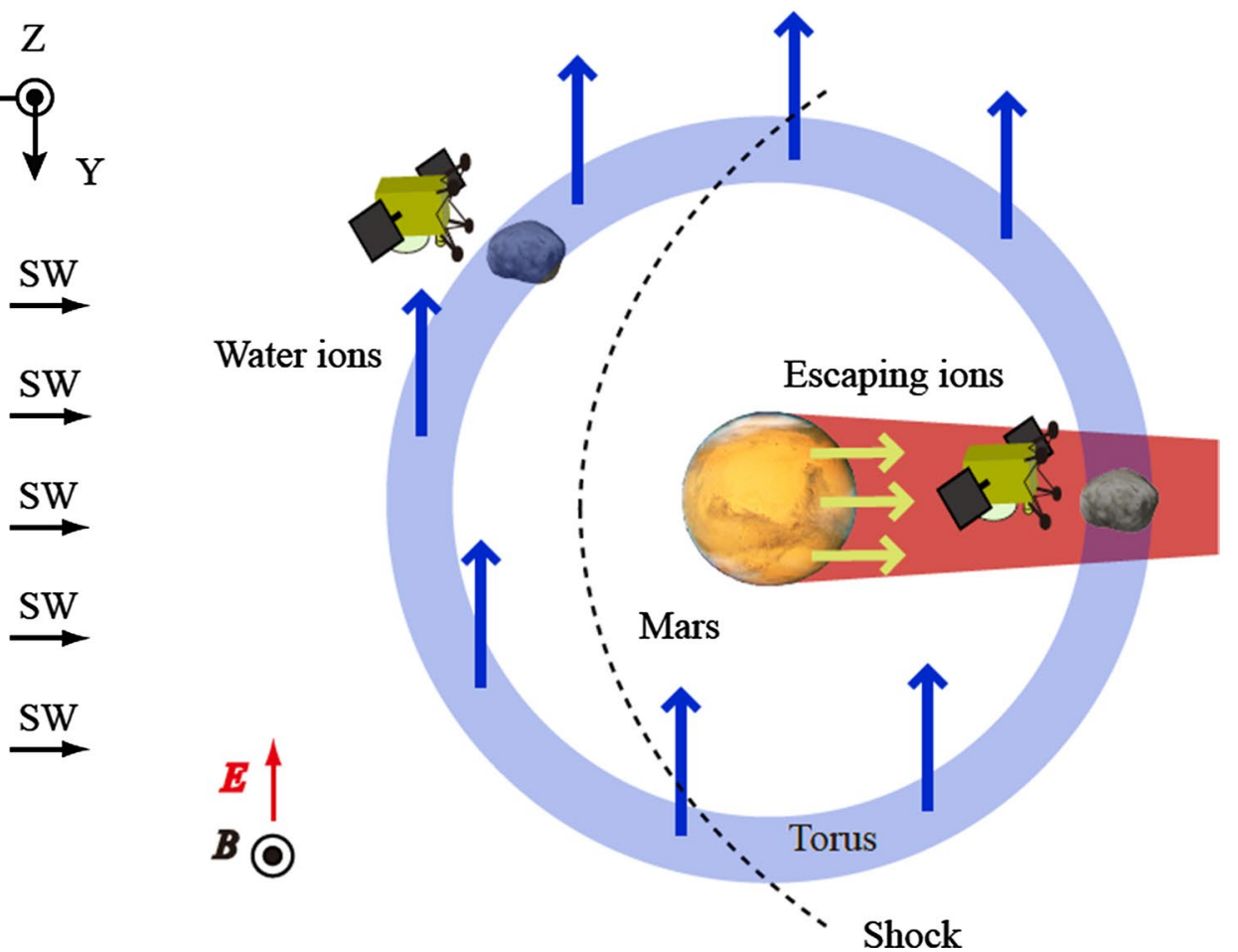

Fig. 5 Observation configurations of MSA. a MSA observations 1 and 3 for measuring solar wind (SW) ions, scattered SW ions on the Phobos surface, and secondary ions sputtered by the SW from Phobos surface. b MSA observations 2 and 4 for measuring water ions from the torus and escaping ions from the Martian atmosphere. In the both panels, $\boldsymbol{B}$ and $\boldsymbol{E}$ indicate the magnetic and electric fields of the SW, respectively. The magnetometer measures $\boldsymbol{B}$, while $E$ is derived from $\boldsymbol{E}=-\boldsymbol{V} \times \boldsymbol{B}$, where $\boldsymbol{V}$ denote the SW velocity measured by the ion analyzer. The Phobos/ Mars-centric Solar Ecliptic coordinate system is used in panels $\mathbf{a}$ and $\mathbf{b}$ 
Mars with respect to the Sun at an interval of $7.66 \mathrm{~h}$, there is an opportunity to observe escaping ions from the Martian atmosphere. It should be noted that there is a Martian corona containing oxygen that extends beyond Phobos orbit as an ion source (Rahmati et al. 2017). To investigate the origin of the observed ions, it is important to consider the motion of the ions based on magnetic field observations, as described next.

The motion of charged particles is determined by the surrounding electric and magnetic fields $(\boldsymbol{E}$ and $\boldsymbol{B})$ in space. The equation of motion in $\boldsymbol{E}$ and $\boldsymbol{B}$ is expressed as $m \dot{v}=q(\boldsymbol{E}+\boldsymbol{v} \times \boldsymbol{B})$, where $m, q$, and $v$ denote the mass, charge, and velocity of the charged particle, respectively. Thus, the information of $\boldsymbol{E}$ and $\boldsymbol{B}$ is indispensable for an adequate interpretation of the ion behavior measured by MSA. In the Martian environment, the solar wind electric and magnetic fields are dominant upstream from
Mars, while the shocked and tail regions are formed downstream. In the solar wind, MSA can derive both $\boldsymbol{E}$ and $\boldsymbol{B}$ from its measurements, because the solar wind electric field is given by $\boldsymbol{E}=-\boldsymbol{V} \times \boldsymbol{B}$, where $\boldsymbol{V}$ indicates the solar wind velocity measured by the MSA ion analyzer.

As a reference for the ion observations around Phobos, the observation results around the Moon can be used, obtained by IMA on the Kaguya spacecraft (Saito et al. 2010) (see Fig. 6). The energy-time spectrogram obtained by IMA shows that solar wind ions, those scattered at the lunar surface and reflected by the lunar magnetic anomalies, and secondary ions emitted from the Moon were measured in a couple of hours. Since the spacecraft was nadir-pointing, IMA measured solar wind ions only around the pole regions, while those scattered at the lunar surface were measured over the dayside surface.

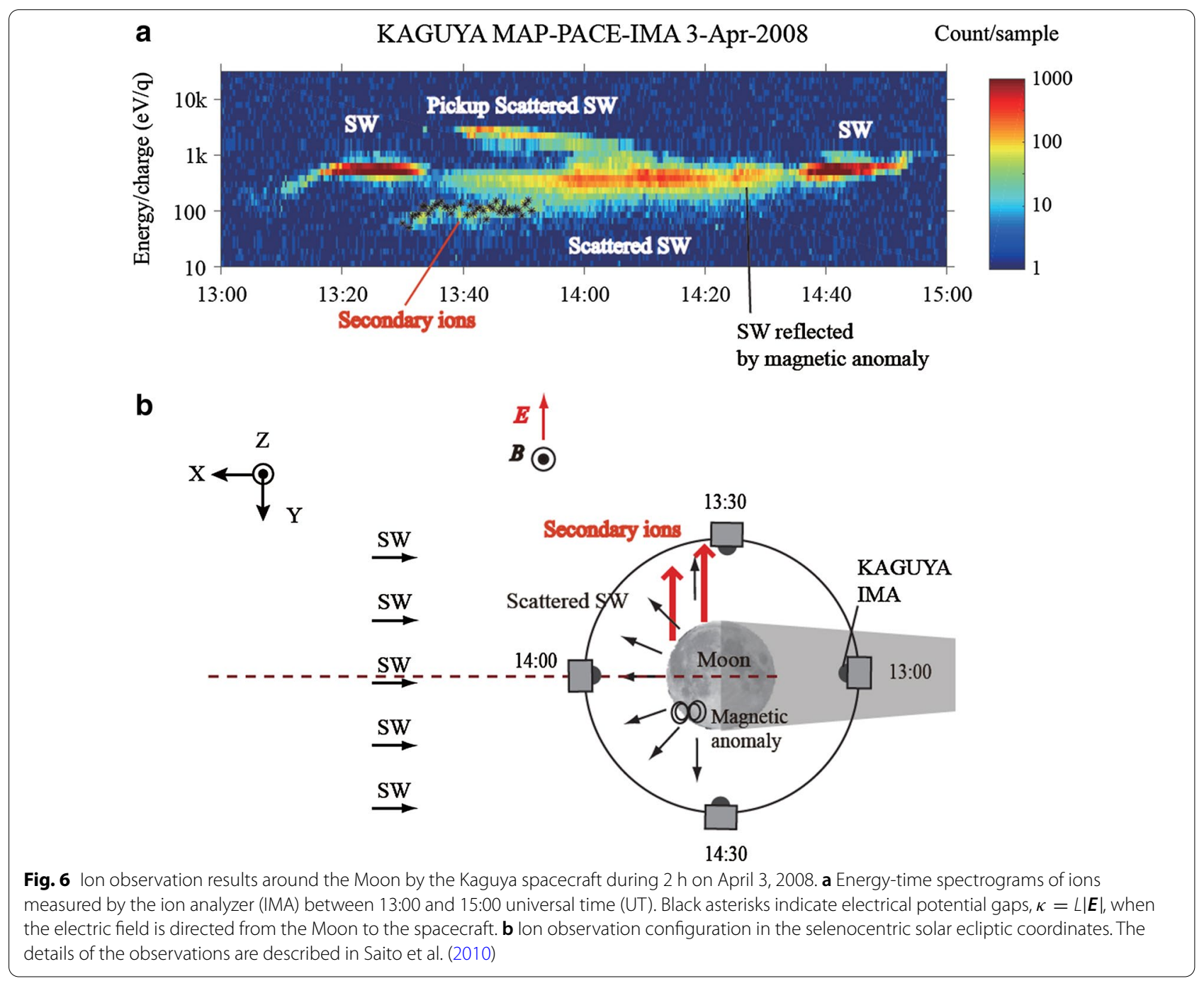


Although the scattered solar wind ions lose $\sim 50 \%$ of their incident energies, they travel against the solar wind for a short distance $(\sim 100 \mathrm{~s} \mathrm{~km})$ (Saito et al. 2008b). Thus, the scattered solar wind ions can be measured around Phobos when the distance is less than $\sim 100 \mathrm{~s} \mathrm{~km}$. On the other hand, the initial energies of secondary ions sputtered by the solar wind are mostly less than a couple of electron volts (Madey et al. 1998 and references therein). Consequently, secondary ions move in a straight direction along $\boldsymbol{E}$ over a short distance $(\sim 100 \mathrm{~s} \mathrm{~km})$ compared to their Larmor radii $(100 \mathrm{~s}-1000 \mathrm{~s} \mathrm{~km})$, while they are in a pickup ion motion due to the solar wind (Yokota and Saito 2005). The motion of the ions generated in the torus is almost the same as that of the secondary ions from Phobos, because their energies in the torus gas before photoionization are less than a few electron volts. Their measured energies $\kappa$ are determined by $\kappa=L|\boldsymbol{E}|$, where $L$ indicates the distance between the emission/ionization points of the ions and spacecraft positions (see Fig. 5a).
Different from the observations of the solar wind ions and those scattered at the surface, the observation of pickup ions generated from the torus and Phobos considerably depends on $\boldsymbol{E}$. Since the torus is widely distributed and is continuously tracked by the spacecraft, MSA has many opportunities to observe ions from the torus. However, the observation of secondary ions emitted from Phobos with a diameter of $d \sim 22.5 \mathrm{~km}$ (MSA observation 1 ) is comparatively limited. A requirement of the MSA observation 1 is that the positions of the spacecraft and Phobos are in the same plane whose normal vector is parallel to $V$ (see Fig. 7). In other words, the spacecraft is in the $Y Z$ plane in the Phobos-centric Solar Ecliptic coordinates because $\boldsymbol{V}$ is approximately on the $X$-axis. During the nominal observation, the spacecraft will periodically stay in the dayside and nightside of Phobos and thus will pass through the $Y Z$ plane many times (Nakamura et al. 2021). In addition, MSA observation 1 has another requirement that $\boldsymbol{E}$ directs from Phobos to the spacecraft. Assuming the direction of $\boldsymbol{B}$ is non-biased, the possibility

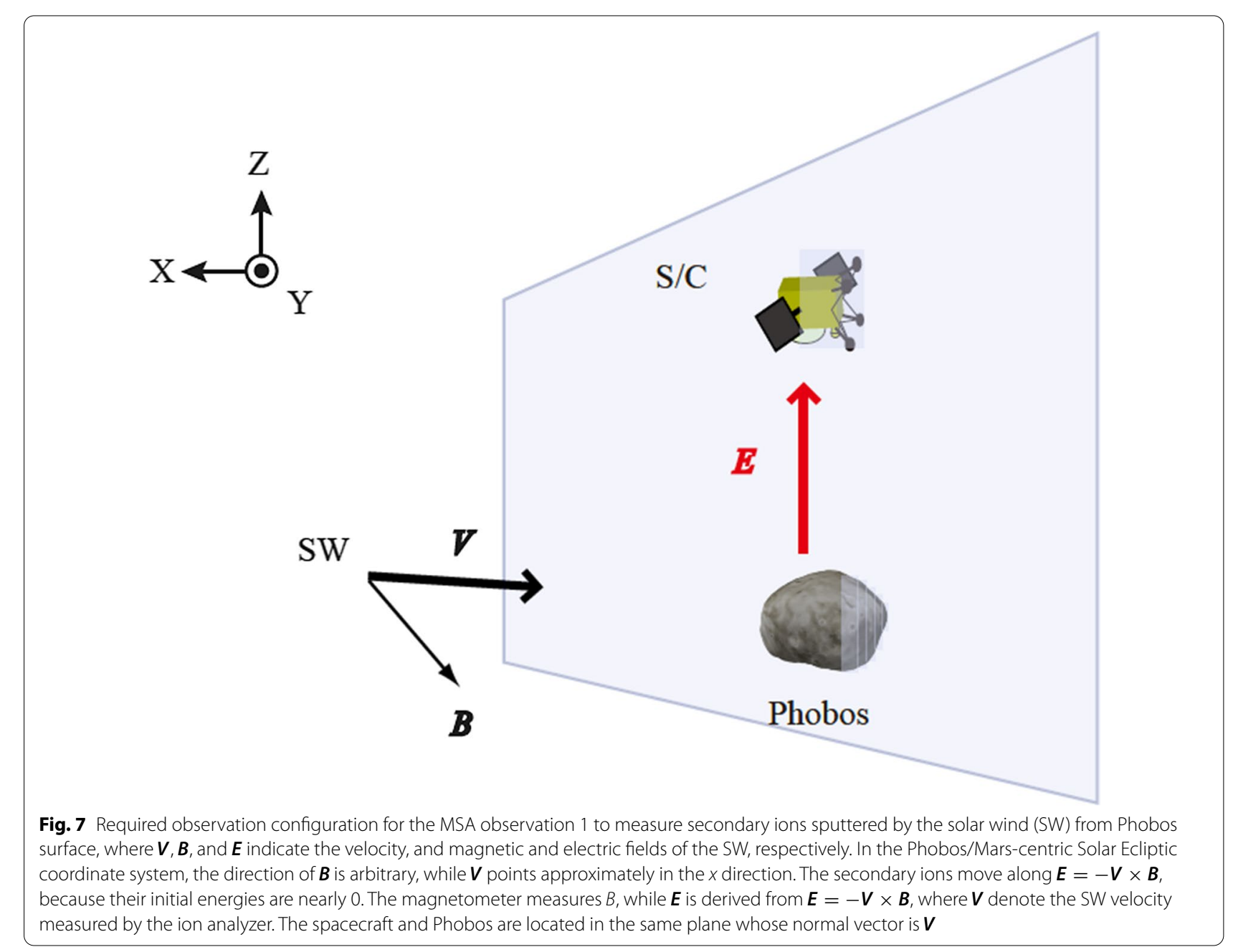


is approximately given by $\frac{d}{2 \pi L}$ for spacecraft in the $Y Z$ plane, where $d$ and $L$ denote the diameter of Phobos and spacecraft's altitude, respectively. Although high-altitude $(\sim 100 \mathrm{~km})$ observations provide the possibility below $5 \%$, the observation period for secondary ions from Phobos will be secured by low-altitude $(10-30 \mathrm{~km})$ observations with the possibility of $10 \mathrm{~s} \%$.

It should be noted that if Phobos had large and widearea magnetic anomalies sufficiently to capture secondary ions, MSA would measure no ions from Phobos. In the case of the Earth's Moon, Kaguya observed solar wind ions reflected from magnetic anomalies rather than secondary ions from the lunar surface (see Fig. 6). In such an extreme case, the MSA magnetometer would tell us what disturbs the MSA ion observation.

\section{MSA magnetic field observation}

As described in the previous subsection, magnetic field vector $\boldsymbol{B}$ is indispensable to interpret the ion behavior measured by the MSA ion energy mass spectrometer. The MSA magnetometer is required to measure the magnetic field strength and direction with good accuracy.

The magnetic field strength in the solar wind at the Mars orbit is typically $3 \mathrm{nT}$, although it significantly varies depending on the condition of the solar surface. Disturbing fields from other components on the spacecraft would cause problems to determine the strength and direction of such weak magnetic field. In the previous missions measuring weak magnetic field in the space, very often the magnetometer sensors have been mounted at the tips of the long booms to avoid the disturbances. In the case of MMX where boom is not available, however, the disturbing noises are planned to be identified quantitatively by analyzing the output values from two magnetometers. At two separated measurement positions on the surface of the spacecraft, the artificial magnetic fields caused by the onboard components have different intensity and direction, while the natural magnetic fields are the same. The magnetic field data are able to be 'cleaned' by analyzing the difference between the fields measured by two magnetometers. Similar method has been used in the missions where it is difficult to implement boom for magnetometer or carry magnetic cleanliness of the spacecraft (Georgescu et al. 2008; Pope et al. 2011; Constantinesc et al. 2020; Imajo et al. 2021).

In the orbit, the MSA magnetometer produces the magnetic field data with $1 \mathrm{~Hz}$ sampling rate. Besides, it has $128 \mathrm{~Hz}$ sampling mode for the instrument checkout. It is much desirable to operate the MSA magnetometer during the cruise to Mars for the purposes of the instrument health check as well as for the scientific study of the interplanetary plasma physics.
The MSA magnetometer will be operated basically together with the MSA ion energy mass spectrometer. On the other hand, even without the data from the ion energy mass spectrometer, it is much valuable to measure the magnetic fields, especially at the landing operation to Phobos. The intrinsic and crustal magnetic fields of Phobos are essential to interpret the interior structure and surface composition. Although there is no reliable information for deriving the magnetic moment of Phobos so far (Veselovsky 2004), Mordovskaya et al. (2001) estimated the magnetic field intensity at the Phobos surface to be 0.6G (same intensity as geomagnetic surface field) from the magnetopause position identified by Phobos-2. Considering the possibility that the magnetic field originates from the magnetic materials contained by Phobos, their analysis estimated the magnetization of the Phobos substance as $150 \mathrm{~A} / \mathrm{m}$. Even if the magnetization is assumed to be much less intense, where Phobos is a sphere with a radius of $10 \mathrm{~km}$ radius homogeneously magnetized at $0.4 \mathrm{~A} / \mathrm{m}$, the magnetic field intensity is more than $3 \mathrm{nT}$, the typical intensity of the magnetic field in the solar wind at the Martian orbit, at distances shorter than $30 \mathrm{~km}$ from the surface. The magnetic field, if detected, would give important insights into the global magnetism of Mars and its history.

The time variation of the magnetic field originating from the Phobos interior can be an indicator of the electrical current flowing under the surface induced by the variation in the external magnetic field. Observations of the time variation would allow us to estimate the electrical conductivity of the material from the surface to the skin depth, and thus provide important information about the crustal material and its condition. Similar observations were carried out by the Apollo Moon missions (Hood et al. 1982), and is planned in the Jupiter JUICE mission to investigate a subsurface ocean (Grasset et al. 2013).

\section{Summary}

The preliminary design phase for MSA was completed in October 2020. The development of an engineering model of the MSA instrument has initiated, aiming the final delivery in 2023 and launch of the MMX spacecraft in 2024. To address the MMX mission goals, MSA will perform in situ observations of ions and magnetic field, which are related with the surface and tori of the Martian moons, and Martian atmosphere, and the solar wind. In addition, MSA will also participate in a joint Mars observation program which is currently being defined (e.g., Ogohara et al. 2021). 


\begin{abstract}
Abbreviations
AC: Alternating current; AU: Astronomical Unit; CPU: Central Processing Unit; DC: Direct current; FM-OFG: Fundamental mode orthogonal fluxgate; FOV: Field-of-view; FPGA: Field-Programmable Gate Array; HVPS: High-voltage power supply; IMA: Ion mass analyzer; JAXA: Japan Aerospace Exploration Agency; LEF: Linear-electric field; MCP: Micro-channel plate; MMX: Martian Moons eXploration; MO: Mission objective; MSA: Mass spectrum analyzer; SIMS: Secondary ion mass spectrometry; TOF: Time-of-flight.
\end{abstract}

\section{Acknowledgements}

The authors would like to express sincere thanks to all members of the MMX mission for their supports. The MSA components are now being manufactured by Meisei Electric Co., Ltd. The ion optics is machined by YS design. The magnetometer sensors are now being manufactured by Tierra Tecnica Ltd.

\section{Authors' contributions}

SY, NT, AM, NM, and MJS wrote the manuscript. SY, YS, DD, KA, and SK contributed to the development of the ion analyzer. AM, NM, and RM contributed to the development of the magnetometer. NT, KS, YF, HN, MNN, KK, and YH contributed to consideration of the observation objectives. SI contributed to the magnetic field data analysis plan. All authors read and approved the final manuscript.

\section{Funding}

This research was partially supported by a Grant-in-Aid for Scientific Research from the Japan Society for the Promotion of Science (\#20K04039, \#17H01164, \#17H02970).

\section{Availability of data and materials}

The data and materials used in this research are available on request to the corresponding author, Dr. Shoichiro Yokota (yokota@ess.sci.osaka-u.ac.jp).

\section{Declarations}

\section{Ethics approval and consent to participate}

Not applicable.

\section{Consent for publication}

Not applicable.

\section{Competing interests}

The authors declare that they have no competing interests.

\section{Author details}

'Osaka University, Machikaneyama-cho, Toyonaka 560-0043, Japan. ${ }^{2}$ Tohoku University, Aoba-ku, Sendai 980-8578, Japan. ${ }^{3}$ Kyoto University, Kitashirakawaoiwake-cho, Sakyo-ku, Kyoto 606-8502, Japan. ${ }^{4}$ Japan Aerospace Exploration Agency, Tsukuba, Ibaraki 305-8505, Japan. ${ }^{5}$ Institute of Space and Astronautical Science, Japan Aerospace Exploration Agency, Yoshinodai, Chuo-ku, Sagamihara 252-5210, Japan. ${ }^{6}$ LPP-CNRS-Sorbonne Universite-Ecole Polytechnique, 4 place Jussieu, 75252 Paris, France. ${ }^{7}$ Swedish Institute of Space Physics, Box 812, 98128 Kiruna, Sweden. ${ }^{8}$ The University of Tokyo, Hongo, Bunkyo-ku 113-0033, Japan. ${ }^{9}$ Georgia Institute of Technology, 901 Atlantic Drive, Atlanta, GA 30332, USA.

Received: 10 December 2020 Accepted: 5 June 2021

Published online: 13 December 2021

\section{References}

Agee CB et al (2013) Unique meteorite from early Amazonian Mars: water-rich basaltic breccia Northwest Africa 7034. Science 339:780-785. https:// doi.org/10.1126/science.1228858

Albee AL, Arvidson RE, Palluconi F, Thorpe T (2001) Overview of the mars global surveyor mission. J Geophys Res Planets 106(E10):23291-23316. https://doi.org/10.1029/2000JE001306

Asamura K, Kazama Y, Yokota S, Kasahara S, Miyoshi Y (2018) Low-energy particle experiments -ion analyzer (LEPi) onboard the ERG (Arase) satellite. Earth Planets Space 70:70. https://doi.org/10.1186/s40623-018-0846-0
Bamford RA et al (2016) 3D PIC simulations of collisionless shocks at lunar magnetic anomalies and their role in forming lunar swirls. Astrophys J 830:146. https://doi.org/10.3847/0004-637X/830/2/146

Barabash $S$ et al (2004) ASPERA-3: analyser of space plasmas and energetic ions for Mars express. In: Wilson A (ed) Mars express: the scientific payload. ESA Special Publication SP-1240. ESA Publications Division, Noordwijk, pp 121-139

Barabash S, Fedorov A, Lundin R, Sauvaud J-A (2007) Martian atmospheric erosion rates. Science 315:501-503. https://doi.org/10.1126/science. 1134358

Baumgärtel K, Sauer K, Dubinin E, Tarrasov V, Dougherty M (1998) "Phobos events" - signatures of solar wind interaction with a gas torus? Earth Planets Space 50:453-462. https://doi.org/10.1186/BF03352133

Baumjohann W et al (2020) The BepiColombo-mio magnetometer en route to mercury. Space Sci Rev 216(8):125

Benna M, Mahaffy PR, Grebowsky JM, Fox JL, Yelle RV, Jakosky BM (2015) First measurements of composition and dynamics of the Martian ionosphere by MAVEN's neutral gas and ion mass spectrometer. Geophys Res Lett 42(21):8958-8965. https://doi.org/10.1002/2015GL066146

Borg LE et al (2005) Constraints on the U-Pb isotopic systematics of Mars inferred from a combined $\mathrm{U}-\mathrm{Pb}, \mathrm{Rb}-\mathrm{Sr}$, and $\mathrm{Sm}-\mathrm{Nd}$ isotopic study of the Martian meteorite Zagami. Geochim Cosmochim Acta 69:58195830. https://doi.org/10.1016/j.gca.2005.08.007

Borin P, Cremonese G, Marzari F, Lucchetti A (2017) Asteroidal and cometary dust flux in the inner solar system. Astron Astrophys 605:A94. https:// doi.org/10.1051/0004-6361/201730617

Brain DA et al (2015) The spatial distribution of planetary ion fluxes near Mars observed by MAVEN. Geophys Res Lett 42:9142-9148. https://doi.org/ 10.1002/2015GL065293

Canup RM, Asphang E (2001) Origin of the Moon in a giant impact near the end of the Earth's formation. Nature 412:708-712

Carlson CW, Curtis DW, Paschmann G, Michel W (1982) An instrument for rapidly measuring plasma distribution functions with high resolution. Adv Space Res 2(7):67-70

Chapman CR (2004) Space weathering of asteroid surfaces. Ann Rev Earth Planet Sci 32:539-567. https://doi.org/10.1146/annurev.earth.32. 101802.120453

Chassefiere E, Leblanc F (2004) Mars atmospheric escape and evolution; interaction with the solar wind. Planet Space Sci 52(11):1039-1058. https:// doi.org/10.1016/j.pss.2004.07.002

Cipriani F, Witasse O, Leblanc F, Modolo R, Johnson RE (2011) A model of interaction of Phobos' surface with the Martian environment. Icarus 212(2):643-648. https://doi.org/10.1016/j.icarus.2011.01.036

Constantinescu OD et al (2020) Principal component gradiometer technique for removal of spacecraft-generated disturbances from magnetic field data. Geosci Instrum Method Data Syst Discuss 2020:1-26

Delcourt D et al (2009) The mass spectrum analyzer (MSA) onboard BEPI COLOMBO MMO: scientific objectives and prototype results. Adv Space Res 43:869-874. https://doi.org/10.1016/j.asr.2008.12.002

Delcourt D et al (2016) The mass spectrum analyzer (MSA) on board the BepiColombo MMO. J Geophys Res Space Phys 121(7):6749-6762

Dhanya MB, Bhardwaj A, Futaana Y, Barabash S, Wieser M, Holmström M, Wurz $P$ (2017) New suprathermal proton population around the moon: observation by SARA on chandrayaan-1. Geophys Res Lett 44(10):45404548. https://doi.org/10.1002/2017GL072605

Dong $Y$ et al (2015) Strong plume fluxes at Mars observed by MAVEN: an important planetary ion escape channel. Geophys Res Lett 42(21):8942-8950. https://doi.org/10.1002/2015GL065346

Dukes CA, Baragiola RA (2015) The lunar surface-exosphere connection: measurement of secondary-ions from Apollo soils. Icarus 255:51-57. https:// doi.org/10.1016/j.icarus.2014.11.032

Elphic RC, Funsten HO III, Barraclough BL, McComas DJ, Paffett MT, Vaniman DT, Heiken G (1991) Lunar surface composition and solar wind-induced secondary ion mass spectrometry. Geophys Res Lett 11:2165-2168

Fanale FP, Salvail JR (1990) Evolution of the water regime of Phobos. Icarus 88(2):380-395. https://doi.org/10.1016/0019-1035(90)90089-R

Feldman WC, Maurice S, Binder AB, Barraclough BL, Elphic RC, Lawrence DJ (1998) Fluxes of fast and epithermal neutrons from lunar prospector: evidence for water ice at the lunar poles. Science 281 (5382):1496-1500. https://doi.org/10.1126/science.281.5382.1496 
Fox JL, Hac A (2010) Isotope fractionation in the photochemical escape of $\mathrm{O}$ from Mars. Icarus 208:176-191. https://doi.org/10.1016/j.icarus.2010. 01.019

Fujiwara A et al (2006) The rubble-pile asteroid Itokawa as observed by Hayabusa. Science 312:1330-1334

Futaana Y, Barabash S, Holmström M, Fedorov A, Nilsson H, Lundin R, Dubinin E, Fränz M (2010a) Backscattered solar wind protons by Phobos. J Geophys Res 115:A10213. https://doi.org/10.1029/2010JA015486

Futaana Y et al (2010b) Protons in the near-lunar wake observed by the subkeV atom reflection analyzer on board Chandrayaan-1. J Geophys Res 115(A10):10. https://doi.org/10.1029/2010JA015264

Futaana $Y$ et al (2013) Remote energetic neutral atom imaging of electric potential over a lunar magnetic anomaly. Geophys Res Lett 40(2):262266. https://doi.org/10.1002/grl.50135

Georgescu E et al (2008) Modified gradiometer technique applied to double star (TC-1). Adv Space Res 41(10):1579-1584

Gilbert JA, Lundgren RA, Panning MH, Rogacki S, Zurbuchen TH (2010) An optimized three-dimensional linear-electric-field time-of-flight analyzer. Rev Sci Instrum 81(5):053302. https://doi.org/10.1063/1.3429941

Grasset O et al (2013) JUpiter ICy moons Explorer (JUICE): an ESA mission to orbit Ganymede and to characterise the Jupiter system. Planet Space Sci 78:1-21

Grotzinger JP et al (2012) Mars science laboratory mission and science investigation. Space Sci Rev 170:5-56. https://doi.org/10.1007/ s11214-012-9892-2

Halekas S, Poppe AR, Delory GT, Sarantos M, McFadden JP (2013) Using ARTEMIS pickup ion observations to place constraints on the lunar atmosphere. J Geophys Res Planets 118:81-88. https://doi.org/10.1029/ 2012JE004292

Halekas JS et al (2017) Structure, dynamics, and seasonal variability of the Mars-solar wind interaction: MAVEN solar wind ion analyzer inflight performance and science results. J Geophys Res Space Phys 122(1):547-578

Higuchi A, Ida S (2017) Temporary capture of asteroids by an eccentric planet. Astrophys J 153:155

Hood LL et al (1982) The deep lunar electrical conductivity profile: structural and thermal inferences. J Geophys Res Solid Earth 87(B7):5311-5326. https://doi.org/10.1029/JB087iB07p05311

Hyodo R et al (2019) Transport of impact ejecta from Mars to its moons as a means to reveal Martian history. Sci Rep 9:19833. https://doi.org/10. 1038/s41598-019-56139-x

Imajo S, Nose M, Aida M, Matsumoto H, Higashio N, Tokunaga T, Matsuoka A (2021) Signal and noise separation from satellite magnetic field data through independent component analysis: prospect of magnetic measurements without boom and noise source information. J Geophys Res Space Phys 126:e2020JA028790. https://doi.org/10.1029/2020J A028790

Inui S et al (2018) Cold dense ion outflow observed in the Martian-induced magnetotail by MAVEN. Geophys Res Lett 45:5283-5289. https://doi. org/10.1029/2018GL077584

Ip WH, Banaszkiewicz M (1990) On the dust/gas tori of Phobos and Deimos. Geophys Res Lett 17:857-860. https://doi.org/10.1029/GL017i006p 00857

Jakosky BM et al (2015) The Mars atmosphere and volatile evolution (MAVEN) mission. Space Sci Rev 195:3-48. https://doi.org/10.1007/ s11214-015-0139-x

Jakosky BM et al (2017) Mars' atmospheric history derived from upper-atmosphere measurements of 38Ar/36Ar. Science 355(6332):1408-1410. https://doi.org/10.1126/science.aai7721

Johnson R, Baragiola R (1991) Lunar surface: sputtering and secondary ion mass spectrometry. Geophys Res Lett 18(11):2169-2172. https://doi. org/10.1029/91GL02095

Koga F, Sasada I (2003) Low offset orthogonal fluxgate operating in fundamental mode. J Magn Soc Jpn 27:410-413

Kramer GY (2011) Characterization of lunar swirls at Mare Ingenii: a model for space weathering at magnetic anomalies. J Geophys Res Planets 116:E4. https://doi.org/10.1029/2010JE003669

Krymskii AM, Breus TK, Dougherty MK, Southwood DJ, Axford WI (1992) The electromagnetic effects of the solar wind interaction with the Phobos neutral gas halo and dust torus. Planet Space Sci 40(8):1033. https://doi. org/10.1016/0032-0633(92)90032-J
Kuramoto K et al (2021) Martian moons exploration MMX: sample return mission to Phobos elucidating formation processes of habitable planets. Earth Planets Space. https://doi.org/10.1186/s40623-021-01545-7

Lawrence DJ et al (2013) Evidence for water ice near Mercury's north pole from MESSENGER neutron spectrometer measurements. Science 339(6117):292-296. https://doi.org/10.1126/science.1229953

Lawrence DJ et al (2019) Measuring the elemental composition of Phobos: the Mars-Moon Exploration with GAmma rays and NEutrons (MEGANE) investigation for the Martian Moons eXploration (MMX) mission. Earth Space Sci 6:2605-2623. https://doi.org/10.1029/2019EA000811

Li S, Lucey PG, Fraeman AA, Poppe AR, Sun VZ, Hurley DM, Schultz PH (2020) Widespread hematite at high latitudes of the Moon. Sci Adv 6:eaba1940. https://doi.org/10.1126/sciadv.aba1940

Lundin R et al (2004) Solar wind-induced atmospheric erosion at Mars: first results from ASPERA-3 on Mars express. Science 305(5692):1933-1936

Madey TE, Yakshinskiy BV, Ageev VN, Johnson RE (1998) Desorption of alkali atoms and ions from oxide surfaces: relative to the origins of $\mathrm{Na}$ and $\mathrm{K}$ in atmospheres of Mercury and the Moon. J Geophys Res 103(E3):5873-5887

Mahaffy PR et al (2013) Abundance and isotopic composition of gases in the Martian atmosphere from the curiosity rover. Science 341:263-266. https://doi.org/10.1126/science.1237966

Maruyama S, Ebisuzaki T (2017) Origin of the Earth: a proposal of new model called ABEL. Geosci Front 8(2):253-274. https://doi.org/10.1016/j.gsf. 2016.10 .005

Matsumoto T, Harries D, Langenhorst F, Miyake A, Noguchi T (2020) Iron whiskers on asteroid Itokawa indicate sulfide destruction by space weathering. Nat Commun 11:1117. https://doi.org/10.1038/s41467-020-14758-3

Matsuoka A et al (2018) The ARASE (ERG) magnetic field investigation. Earth Planets Space 70:43. https://doi.org/10.1186/s40623-018-0800-1

McComas DJ, Nordholt JE (1990) New approach to 3-D, high sensitivity, high mass resolution space plasma composition measurements. Rev Sci Instrum 61(10):3095. https://doi.org/10.1063/1.114169

McComas DJ, Allegrini F, Bochsler P, Frisch P, Funsten HO, Gruntman M, Janzen PH et al (2009) Lunar backscatter and neutralization of the solar wind: first observations of neutral atoms from the Moon. Geophys Res Lett 36(12):2-5. https://doi.org/10.1029/2009GL038794

McElroy MB, Yung YL (1976) Oxygen isotopes in the Martian atmosphere: implications for the evolution of volatiles. Planet Space Sci 24:11071113. https://doi.org/10.1016/0032-0633(76)90148-3

McFadden JP et al (2015) MAVEN suprathermal and thermal ion composition (STATIC) instrument. Space Sci Rev 195:199-256. https://doi.org/10. 1007/s11214-015-0175-6

Milillo A et al (2020) Investigating Mercury's environment with the two-spacecraft BepiColombo mission. Space Sci Rev 216:93. https://doi.org/10. 1007/s11214-020-00712-8

Miyoshi Y et al (2018) Geospace exploration project ERG. Earth Planets Space 70:101. https://doi.org/10.1186/s40623-018-0862-0

Mordovskaya VG, Oraevsky VN, Styashkin VA (2001) Experimental evidence of the Phobos magnetic field. J Environ Theor Phys Lett 74:293-297. https://doi.org/10.1134/1.1421402

Mortimer J, Verchovsky AB, Anand M (2016) Predominantly non-solar origin of nitrogen in lunar soils. Geochim Cosmochim Acta 193:36-53. https:// doi.org/10.1016/j.gca.2016.08.006

Mura A, Milillo A, Orsini S, Kallio E, Barabash S (2002) Energetic neutral atoms at Mars 2. Imaging of the solar wind-Phobos interaction. J Geophys Res 107(10):1278. https://doi.org/10.1029/2001JA000328

Murata N, Karo H, Sasada I, Shimizu T (2018) Fundamental mode orthogonal fluxgate magnetometer applicable for measurements of DC and lowfrequency magnetic fields. IEEE Sens J 18:2705-2712

Murata N, Nomura R, Matsuoka A (2019) Current annealing of amorphous wire core for performance improvement of fundamental mode orthogonal fluxgate. J Magn Magn Mater 484:497-503

Mutzke A, Schneider R, Eckstein W, Dohmen R, Schmid K, Toussaint UV Badelow G (2011) SDTrimSP version 5.00: IPP-report. Max-Planck-Institut für Plasmaphysik, Garching, Germany, Tech. Rep 12(8)

Nakamura T et al (2021) Science operation plan of Phobos and Deimos from the MMX spacecraft. Earth Planets Space. https://doi.org/10.1186/ s40623-021-01546-6

Nénon Q et al (2019) Phobos surface sputtering as inferred from MAVEN ion observations. J Geophys Res Planets 124:3385-3401 
Nénon Q, Poppe AR, Rahmati A, McFadden JP (2021) Implantation of Martian atmospheric ions within the regolith of Phobos. Nat Geosci 14:61-66. https://doi.org/10.1038/s41561-020-00682-0

Nishino MN et al (2009) Solar-wind proton access deep into the near-Moon wake. Geophys Res Lett. https://doi.org/10.1029/2009GL039444

Nishino MN et al (2010) Effect of the solar wind proton entry into the deepest lunar wake. Geophys Res Lett. https://doi.org/10.1029/2010GL043948

Nishino MN et al (2013) Type-Il entry of solar wind protons into the lunar wake: effects of magnetic connection to the night-side surface. Planet Space Sci 87:106-114. https://doi.org/10.1016/j.pss.2013.08.017

Nishino MN et al (2017) Kaguya observations of the lunar wake in the terrestrial foreshock: surface potential change by bow-shock reflected ions. Icarus 293:45-51. https://doi.org/10.1016/j.icarus.2017.04.005

Nittler LR, McCoy TJ, Clark PE, Murphy ME, Trombka JI, Jarosewich E (2004) Bulk element compositions of meteorites: a guide for interpreting remotesensing geochemical measurements of planets and asteroids. Antarct Meteor Res 17:231-251

Nittler LR et al (2011) The major-element composition of Mercury's surface from MESSENGER X-ray spectrometry. Science 333:1847-1850. https:// doi.org/10.1126/science.1211567

Ogohara K et al (2021) The Mars system revealed by the Martian Moons exploration mission. Earth Planets Space. https://doi.org/10.1186/ s40623-021-01417-0

Øieroset M et al (2010) Search for Phobos and Deimos gas/dust tori using in situ observations from Mars global surveyor MAG/ER. Icarus 206:189-198. https://doi.org/10.1016/j.icarus.2009.07.017

Ozima M, Seki K, Terada N, Miura YN, Podosek FA, Shinagawa H (2005) Terrestrial nitrogen and noble gases in lunar soils. Nature 436:655-659

Ozima M, Yin Q-Z, Podosekc FA, Miura YN (2008) Toward understanding early Earth evolution: prescription for approach from terrestrial noble gas and light element records in lunar soils. Proc Natl Acad Sci 105:17654-17658

Pollock C et al (2016) Fast plasma investigation for magnetospheric multiscale. Space Sci Rev 199(1):331-406. https://doi.org/10.1007/ s11214-016-0245-4

Pope SA et al (2011) Exploring planetary magnetic environments using magnetically unclean spacecraft: a systems approach to VEX MAG data analysis. Ann Geophys 29(4):639-647

Poppe AR, Curry SM (2014) Martian planetary heavy ion sputtering of Phobos. Geophys Res Lett 41(18):6335-6341. https://doi.org/10.1002/2014G L061100

Poppe AR, Curry SM, Fatemi S (2016) The Phobos neutral and ionized torus. J Geophys Res Planets 121(5):770-783. https://doi.org/10.1002/2015 J E004948

Rahmati A (2017) MAVEN measured oxygen and hydrogen pickup ions: probing the Martian exosphere and neutral escape. J Geophys Res Space Phys 122:3689-3706. https://doi.org/10.1002/2016JA023371

Ramstad R, Barabash S, Futaana Y, Nilsson H, Holmström M (2017) Global Marssolar wind coupling and ion escape. J Geophys Res 122(8):8051-8062. https://doi.org/10.1002/2017JA024306

Richmond NC, Hood LL (2008) A preliminary global map of the vector lunar crustal magnetic field based on lunar prospector magnetometer data. J Geophys Res 113:E02010. https://doi.org/10.1029/2007JE002933

Rosenblatt P et al (2016) Accretion of Phobos and Deimos in an extended debris disc stirred by transient moons. Nat Geosci 9:581-583

Saal AE, Hauri EH, Cascio ML, van Orman JA, Rutherford MC, Cooper RF (2008) Volatile content of lunar volcanic glasses and the presence of water in the Moon's interior. Nature 454:192-195. https://doi.org/10.1038/natur e07047

Saito Y et al (2008a) Low energy charged particle measurement by MAP-PACE onboard SELENE. Earth Planets Space 60:375-385. https://doi.org/10. 1186/BF03352802

Saito $Y$ et al (2008b) Solar wind proton reflection at the lunar surface: low energy ion measurement by MAP-PACE onboard SELENE (KAGUYA). Geophys Res Lett 35:L24205. https://doi.org/10.1029/2008GL036077

Saito $Y$ et al (2010) In-flight performance and initial results of plasma energy angle and composition experiment (PACE) on SELENE (Kaguya). Space Sci Rev 154(1-4):265-303. https://doi.org/10.1007/s11214-010-9647-x

Saito $Y$ et al (2012) Simultaneous observation of the electron acceleration and ion deceleration over lunar magnetic anomalies. Earth Planets Space 64:83-92. https://doi.org/10.5047/eps.2011.07.011
Saito Y, Yokota S, Asamura K (2017) High speed MCP anodes for high timeresolution low-energy charged particle spectrometers. J Geophys Res 122(2):1816-1830. https://doi.org/10.1002/2016JA023157

Saito $Y$ et al (2021) Pre-flight calibration and near-earth commissioning results of the Mercury Plasma Particle Experiment (MPPE) onboard MMO (Mio) Space Sci Rev 217:70. https://doi.org/10.1007/s11214-021-00839-2

Sasada I (2002) Orthogonal fluxgate mechanism operated with dc biased excitation. J Appl Phys 91:7789-7791

Sasada I, Usui T (2003) Orthogonal fluxgate magnetometer utilizing bias switching for stable operation. Proc IEEE Sens 2:468-471

Sasaki S, Nakamura K, Hamabe Y, Kurahashi E, Hiroi T (2001) Production of iron nanoparticles by laser irradiation in a simulation of lunar-like space weathering. Nature 410:555-557. https://doi.org/10.1038/35069013

Schaible MJ (2014) The role of solar wind in the formation of hydroxyl on airless silicate bodies in space (Master's thesis), University of Virginia, Charlottesville, VA

Schaible MJ, Dukes CA, Hutcherson AC, Lee P, Collier MR, Johnson RE (2017) Solar wind sputtering rates of small bodies and ion mass spectrometry detection of secondary ions. J Geophys Res Planets 122:1968-1983. https://doi.org/10.1002/2017JE005359

Seki K, Elphic RC, Hirahara M, Terasawa T, Mukai T (2001) On atmospheric loss of oxygen ions from Earth through magnetospheric processes. Science 291:1939-1941

Tanaka T et al (2009) First in situ observation of the Moon-originating ions in the Earth's magnetosphere by MAP-PACE on SELENE (KAGUYA). Geophys Res Lett 36:L22106. https://doi.org/10.1029/2009GL040682

Taylor SR, Taylor GJ, Taylor LA (2006) The moon: a Taylor perspective. Geochim Cosmochim Acta 70:5904-5918

Terada K, Yokota S, Saito Y, Kitamura N, Asamura K, Nishino MN (2017) Biogenic oxygen from Earth transported to the Moon by a wind of magnetospheric ions. Nat Astron 1:0026. https://doi.org/10.1038/ s41550-016-0026

Terada K et al (2018) Thermal and impact histories of 25143 Itokawa recorded in Hayabusa particles. Sci Rep 8:11806. https://doi.org/10.1038/ s41598-018-30192-4

Trotignon JG, Grard R, Barabash S, Lundin R, Dubinin E (1996) Solar wind measurements near Mars and their implication in the Red Planet environment. Planet Space Sci 44(2):117-127

Tsunakawa H, Shibuya H, Takahashi F, Shimizu H, Matsushima M, Matsuoka A, Nakazawa S, Otake H, lijima Y (2010) Lunar magnetic field observation and initial global mapping of lunar magnetic anomalies by MAP-LMAG onboard SELENE (Kaguya). Space Sci Rev 154:219-251

Tsunakawa H, Takahashi F, Shimizu H, Shibuya H, Matsushima M (2015) Surface vector mapping of magnetic anomalies over the Moon using Kaguya and lunar prospector observations. J Geophys Res 120:1160-1185

Usui T et al (2020) The importance of Phobos sample return for understanding the Mars-Moon system. Space Sci Rev 216:49. https://doi.org/10.1007/ s11214-020-00668-9

Vernazza PR, Binzel P, Rossi A, Fulchignoni M, Birlan M (2009) Solar wind as the origin of rapid reddening of asteroid surfaces. Nature 458:993

Veselovsky IS (2004) Is Phobos magnetized? Sol Syst Res 38:188-193

Wang HZ (2021) Earth wind as a possible exogenous source of lunar surface hydration. Astrophys J Lett 907:L32. https://doi.org/10.3847/2041-8213/ abd559

Watanabe $\mathrm{S}$ et al (2019) Hayabusa2 arrives at the carbonaceous asteroid 162173 Ryugu — a spinning top-shaped rubble pile. Science 364:268-272

Wei $Y$ et al (2020) Implantation of Earth's atmospheric ions into the nearside and farside lunar soil: implications to geodynamo evolution. Geophys Res Lett 47(3):e2019GL086208. https://doi.org/10.1029/2019GL086208

Wieser $\mathrm{M}$ et al (2009) Extremely high reflection of solar wind protons as neutral hydrogen atoms from regolith in space. Planet Space Sci 57:2132-2134

Wilson A (ed) (2004) Mars express: the scientific payload. ESA Special Publication SP-1240. ESA Publications Division, Noordwijk

Yokota S, Saito Y (2005) Estimation of picked-up lunar ions for future compositional remote SIMS analyses of the lunar surface. Earth Planets Space 57:281-289. https://doi.org/10.1186/BF03352564

Yokota S, Saito Y, Asamura K, Mukai T (2005) Development of an ion energy mass spectrometer for application on board three-axis stabilized spacecraft. Rev Sci Instrum 76:014501-014508 
Yokota S et al (2009) First direct detection of ions originating from the Moon by MAP-PACE IMA onboard SELENE (KAGUYA). Geophys Res Lett 36:L11201

Yokota S et al (2014) Structure of the ionized lunar sodium and potassium exosphere: dawn-dusk asymmetry. J Geophys Res 119(4):798-809. https:// doi.org/10.1002/2013JE004529

Yokota S, Kasahara S, Mitani T, Asamura K, Hirahara M, Takashima T, Yamamoto K, Shibano Y (2017) Medium-energy particle experiments-ion mass analyzer (MEP-i) onboard ERG (Arase). Earth Planets Space 69:172. https://doi.org/10.1186/s40623-017-0754-8

Yokota S et al (2020) Global emissions of indigenous carbon ions from the Moon. Sci Adv 6:eaba1050. https://doi.org/10.1126/sciadv.aba1050

\section{Publisher's Note}

Springer Nature remains neutral with regard to jurisdictional claims in published maps and institutional affiliations.

\section{Submit your manuscript to a SpringerOpen ${ }^{\circ}$ journal and benefit from:}

- Convenient online submission

- Rigorous peer review

- Open access: articles freely available online

- High visibility within the field

- Retaining the copyright to your article

Submit your next manuscript at $\boldsymbol{\nabla}$ springeropen.com 